\title{
Recursive right-tailed unit root tests for an explosive asset price bubble*
}

\author{
David I. Harvey ${ }^{\dagger}$, Stephen J. Leybourne ${ }^{\dagger}$ and Robert Sollis ${ }^{\dagger \dagger}$ \\ ${ }^{\dagger}$ School of Economics, University of Nottingham \\ ${ }^{\dagger \dagger}$ Newcastle University Business School
}

October 2013

\begin{abstract}
In this paper we compare the local asymptotic and finite sample power of two recently proposed recursive right-tailed Dickey-Fuller-type tests for an explosive rational bubble in asset prices. It is shown that the power of the two tests can differ substantially depending on the location of the explosive regime, and whether such a regime ends in collapse. Since this information is typically unknown to the practitioner, we propose a union of rejections strategy that combines inference from the two individual tests. We find that, for a given specification of the explosive regime, the union of rejections strategy always attains power close to the better of the individual tests considered. An empirical illustration using the Nasdaq composite price index is also provided.
\end{abstract}

Keywords: Rational bubble; Explosive autoregression; Unit root testing.

JEL Classification: C22; C12; G14.

\section{Introduction}

A substantial body of theoretical and empirical research exists on statistically testing for explosive asset price bubbles. Much of this literature has concentrated on the class of "rational" explosive asset price bubbles where, despite the asset being overvalued, it is still rational for investors to buy additional units because of the returns available relative to the risk-free discount rate. Orthodox financial theory suggests that the presence of explosive rational asset price bubbles should be relatively easy to uncover

${ }^{*}$ Correspondence to: Robert Sollis, Newcastle University Business School, Newcastle University, 5 Barrack Road, Newcastle upon Tyne, NE1 4SE, United Kingdom. Email: robert.sollis@ncl.ac.uk 
using simple econometric techniques. Consider for example the standard present value model for the fundamental price of a stock $P_{t}^{f}$

$$
P_{t}^{f}=\sum_{i=1}^{\infty}(1+r)^{-i} E_{t}\left(D_{t+i}\right)
$$

where $D_{t}$ denotes the dividend and $r$ denotes the risk-free discount rate. If the transversality condition $\lim _{n \rightarrow \infty} E_{t}\left[(1+r)^{-n} P_{t+n}\right]=0$ holds, then it can be shown via the standard no arbitrage condition that the current price of the stock $P_{t}$ will be equal to the fundamental price $P_{t}^{f}$. However if the transversality condition above does not hold an explosive rational bubble can exist and the price can be decomposed into the fundamentals component $P_{t}^{f}$ and a bubble component $B_{t}$, i.e.

$$
P_{t}=P_{t}^{f}+B_{t}
$$

where $B_{t}$ grows with an expected growth rate equal to $r$. There are infinitely many models for $B_{t}$ that satisfy this condition and so a definitive test for the presence of a particular type of rational asset price bubble is not feasible. However, it follows straightforwardly from the representation above that if an asset price is not more explosive than the fundamentals component of the price, then a bubble does not exist.

In seminal work on this issue, drawing on the fact that an explosive series is also explosive in first differences, Diba and Grossman (1988) proposed testing the hypothesis of no explosive rational asset price bubble using standard left-tailed Dickey-Fuller (DF) unit root tests applied to stock price and dividends series in levels and first differences, with the absence of an explosive rational bubble inferred from a finding of stationarity in the first differences of stock prices.

More direct approaches to identifying explosive rational bubbles have subsequently been proposed, using right-tailed DF tests to detect explosive autoregressive behaviour in stock price series. Hall et al. (1999) initially considered an approach based on Markov-switching autoregressive models, but more recent work in this area has focused on using recursive DF-type tests. Specifically, Phillips et al. (2011) (PWY) suggest detecting explosive rational bubbles using the supremum of a set of forward recursive DF tests applied to the asset price and the relevant fundamentals series, while Homm and Breitung (2012) (HB) recommend using the supremum of a set of backward recursive (DF-type) Chow tests for a change from unit root to explosive autoregressive behaviour. PWY subsequently advocate using their forward recursive statistics to construct a method for time-stamping the start and end dates of the explosive regime; HB suggest an alternative method for dating the origination of explosive behaviour based on their backward recursive approach.

In this paper we analyze the relative local asymptotic and finite sample power performance of the PWY and HB tests to detect explosive autoregressive behaviour. The maintained hypothesis for the data is a random walk process, while our alternative hypotheses variously allow for different timings and durations of a single explosive period. Since any bubble in stock prices that originates during the sample period under study may also terminate prior to the end of the sample, possibly with some 
form of collapse (see, for example, Evans, 1991), we include DGP designs that allow for such behaviour. Specifically, in addition to explosive periods that run up to the end of the sample, we consider non-collapsing explosive periods that return to random walk behaviour at some point prior to the sample's end, and also cases where the postexplosive random walk regime is re-initialized at its pre-explosive level, modelling a bubble that terminates with instantaneous collapse.

The results from our asymptotic and finite sample simulations are important for practitioners as they reveal that the relative performance of the tests can differ quite dramatically depending on the location and timing of the explosive regime, and also whether or not the explosive period terminates in collapse. Overall, we find that the PWY test is better suited to detecting explosive regimes than the HB test when the period of explosiveness occurs early or towards the middle of the sample, while the HB test is better when this regime occurs towards the end of the sample, provided the explosive period does not end in collapse. These results raise the interesting possibility that when the timing of the bubble is unknown, as it would be in practice, a composite test based on a union of rejections strategy applied to the PWY and HB test statistics could yield benefits to practitioners relative to either of these tests being used individually. This type of strategy, based on rejecting the null hypothesis if any of a number of individual tests indicate rejection, has previously been employed in the literature on testing for a unit root against a stationary alternative, for example when uncertainty exists regarding the presence of a trend in the data, or when uncertainty surrounds the nature of the initial value of the series (see Harvey et al., 2009, 2012). We propose such a strategy involving the PWY and HB tests which is asymptotically correctly sized under the null hypothesis, and compare its power performance with that of the individual tests using local asymptotic and finite sample simulations. We find that, for a given specification of the explosive regime, the union of rejections approach displays power close to the better of the individual PWY and HB tests.

The next section of the paper briefly outlines the original PWY and HB tests. Section 3 presents our model, derives the local asymptotic distributions of the tests, and discusses the results from our asymptotic simulations to assess the power of the original tests when explosive periods of varying timings and durations are present. Section 4 reports the findings of our finite sample simulations, where a close correspondence to the asymptotic results is seen. Section 5 details the union of rejections strategy proposed and evaluates the asymptotic and finite sample power of this approach relative to that of the individual PWY and HB tests. Section 6 applies the tests to the Nasdaq composite price index, and Section 7 concludes. The following notation is used: ' $\lfloor\cdot\rfloor$ ' denotes the integer part, ' $\stackrel{d}{\rightarrow}$ ' denotes weak convergence, ' $\stackrel{p}{\rightarrow}$ ' denotes convergence in probability, and $\mathbb{I}($.$) denotes the indicator function.$

\section{Recursive right-tailed unit root tests}

Consider an observed time series $y_{t}, t=1, \ldots, T$, where our interest focuses on testing the null that $y_{t}$ follows a unit root $\operatorname{AR}(1)$ process throughout the full sample, against the alternative that $y_{t}$ behaves as an explosive $\mathrm{AR}(1)$ process for at least some sub- 
period of the sample. In this context, and in the absence of knowledge concerning the timing of any potential explosive behaviour, PWY propose a test based on the supremum of recursive right-tailed DF tests. Specifically, the test statistic is given by

$$
P W Y=\sup _{\tau \in\left[\tau_{0}, 1\right]} D F_{\tau}
$$

where $D F_{\tau}$ denotes the standard DF test, that is the $t$-ratio on $\hat{\phi}$ in the fitted ordinary least squares (OLS) regression

$$
\Delta y_{t}=\hat{\alpha}+\hat{\phi}_{P W Y} y_{t-1}+\hat{\varepsilon}_{t}
$$

calculated over the sub-sample period $t=1, \ldots,\lfloor\tau T\rfloor$, i.e.

$$
D F_{\tau}=\frac{\hat{\phi}_{P W Y}}{\sqrt{\hat{\sigma}_{P W Y}^{2} / \sum_{t=2}^{\lfloor\tau T\rfloor}\left(y_{t-1}-\bar{y}_{\tau}\right)^{2}}}
$$

where $\bar{y}_{\tau}=(\lfloor\tau T\rfloor-1)^{-1} \sum_{t=2}^{\lfloor\tau T\rfloor} y_{t-1}$ and $\hat{\sigma}_{P W Y}^{2}=(\lfloor\tau T\rfloor-3)^{-1} \sum_{t=2}^{\lfloor\tau T\rfloor} \hat{\varepsilon}_{t}^{2}$. The $P W Y$ statistic is therefore the supremum of a sequence of forward recursive statistics with minimum sample length $\left\lfloor\tau_{0} T\right\rfloor$.

HB propose an alternative approach, based on the supremum of recursive Chow tests. Assuming a structure for the alternative hypothesis that specifies $y_{t}$ as a unit root process up to some change-point $\lfloor\tau T\rfloor$, and explosive thereafter, they consider structural change tests based on the fitted full-sample OLS regression

$$
\Delta \tilde{y}_{t}=\hat{\phi}_{H B} \mathbb{I}(t>\lfloor\tau T\rfloor) \tilde{y}_{t-1}+\hat{e}_{t}
$$

where, in the case where the series is permitted to have a non-zero mean, $\tilde{y}_{t}=y_{t}-\bar{y}$ (i.e. full-sample OLS demeaned). The Chow (DF-type) statistic in this setting is given by the $t$-ratio on $\hat{\phi}_{H B}$, which we denote by $C_{\tau}$ :

$$
C_{\tau}=\frac{\hat{\phi}_{H B}}{\sqrt{\hat{\sigma}_{H B}^{2} / \sum_{t=\lfloor\tau T\rfloor+1}^{T} \tilde{y}_{t-1}^{2}}}
$$

where $\hat{\sigma}_{H B}^{2}=(T-2)^{-1} \sum_{t=2}^{T} \hat{e}_{t}^{2}$. HB then propose the test statistic

$$
H B=\sup _{\tau \in\left[0,1-\tau_{0}\right]} C_{\tau}
$$

i.e. the supremum of a sequence of backward recursive statistics, with minimum potential explosive regime length $\left\lfloor\tau_{0} T\right\rfloor$. Both the $P W Y$ and $H B$ statistics can be adjusted to account for additional serial correlation in $y_{t}$ via the usual lagged difference augmentation to the DF-type regressions (1) and (2). 


\section{Model and asymptotic results}

To evaluate the performance of $P W Y$ and $H B$ in detecting explosive, and potentially collapsing, bubble behaviour, we will consider the following model

$$
y_{t}= \begin{cases}y_{t-1}+v_{t} & t=2, \ldots,\left\lfloor\tau_{1} T\right\rfloor \\ (1+\delta) y_{t-1}+v_{t} & t=\left\lfloor\tau_{1} T+1\right\rfloor, \ldots,\left\lfloor\tau_{2} T\right\rfloor \\ y_{t-1}+v_{t} & t=\left\lfloor\tau_{2} T+2\right\rfloor, \ldots, T\end{cases}
$$

with $\delta \geq 0, y_{1}=v_{1}$ and $y_{\left\lfloor\tau_{2} T\right\rfloor+1}=y_{\left\lfloor\tau_{2} T\right\rfloor}+v_{\left\lfloor\tau_{2} T\right\rfloor+1}+y^{*} \mathbb{I}(\delta>0)$. Here, $v_{t}$ is assumed to follow a martingale difference sequence with conditional variance $\sigma^{2}$ and $\sup _{t} E\left(\varepsilon_{t}^{4}\right)<\infty$, with $v_{1}=o_{p}\left(T^{-1 / 2}\right)$.

This DGP imposes a unit root on $y_{t}$ up to time $\left\lfloor\tau_{1} T\right\rfloor$, after which $y_{t}$ is explosive when $\delta>0$ until time $\left\lfloor\tau_{2} T\right\rfloor$. In the third regime, the series reverts to unit root behaviour, and we consider two specifications for the initialization of this latter regime: Case 1. $y^{*}=0$, so that the unit root process is initialized at the last value of the explosive regime; and Case 2. $y^{*}=y_{\left\lfloor\tau_{1} T\right\rfloor}-y_{\left\lfloor\tau_{2} T\right\rfloor}$, where the final unit root regime is initialized at the point prior to the explosive period, modelling the case where the explosive bubble collapses and the level of the series reverts to its pre-explosive value, cf. equation (14) in PWY and the subsequent discussion therein. (Note that the $y^{*}$ adjustment plays no role when $\delta=0$.) We define the null and alternative hypotheses $H_{0}: \delta=0$ and $H_{1}: \delta>0$.

For local alternative hypotheses of the form $\delta=c / T, c>0$, the following Theorem gives the asymptotic properties of $P W Y$ and $H B$.

\section{Theorem 1}

(i) For Case 1,

$$
\begin{aligned}
& P W Y \stackrel{d}{\rightarrow} \sup _{\tau \in\left[\tau_{0}, 1\right]} \frac{\int_{0}^{\tau} \tilde{K}_{c, \tau_{1}, \tau_{2}}(r) d K_{c, \tau_{1}, \tau_{2}}(r)}{\sqrt{\int_{0}^{\tau} \tilde{K}_{c, \tau_{1}, \tau_{2}}(r)^{2} d r}} \equiv L_{c, \tau_{1}, \tau_{2}}^{P W Y}, \\
& H B \stackrel{d}{\rightarrow} \sup _{\tau \in\left[0,1-\tau_{0}\right]} \frac{\int_{\tau}^{1} \bar{K}_{c, \tau_{1}, \tau_{2}}(r) d K_{c, \tau_{1}, \tau_{2}}(r)}{\sqrt{\int_{\tau}^{1} \bar{K}_{c, \tau_{1}, \tau_{2}}(r)^{2} d r}} \equiv L_{c, \tau_{1}, \tau_{2}}^{H B}
\end{aligned}
$$

where

$$
\begin{aligned}
\tilde{K}_{c, \tau_{1}, \tau_{2}}(r) & =K_{c, \tau_{1}, \tau_{2}}(r)-\frac{1}{r} \int_{0}^{r} K_{c, \tau_{1}, \tau_{2}}(s) d s \\
\bar{K}_{c, \tau_{1}, \tau_{2}}(r) & =K_{c, \tau_{1}, \tau_{2}}(r)-\int_{0}^{1} K_{c, \tau_{1}, \tau_{2}}(s) d s
\end{aligned}
$$

with

$$
K_{c, \tau_{1}, \tau_{2}}(r)= \begin{cases}W(r) & r \leq \tau_{1} \\ e^{\left(r-\tau_{1}\right) c} W\left(\tau_{1}\right)+\int_{\tau_{1}}^{r} e^{(r-s) c} d W(s) & \tau_{1}<r \leq \tau_{2} \\ e^{\left(\tau_{2}-\tau_{1}\right) c} W\left(\tau_{1}\right)+\int_{\tau_{1}}^{\tau_{2}} e^{\left(\tau_{2}-s\right) c} d W(s)+W(r)-W\left(\tau_{2}\right) & r>\tau_{2}\end{cases}
$$

and $W(r)$ a standard Brownian motion process. 
(ii) For Case 2,

$$
\begin{aligned}
P W Y & \stackrel{d}{\rightarrow} \sup _{\tau \in\left[\tau_{0}, 1\right]} \frac{\int_{0}^{\tau} \tilde{K}_{c, \tau_{1}, \tau_{2}}^{*}(r) d K_{c, \tau_{1}, \tau_{2}}^{*}(r)}{\sqrt{\pi_{c, \tau_{1}, \tau_{2}}(\tau) \int_{0}^{\tau} \tilde{K}_{c, \tau_{1}, \tau_{2}}^{*}(r)^{2} d r}} \equiv L_{c, \tau_{1}, \tau_{2}}^{* P W Y}, \\
H B \stackrel{d}{\rightarrow} & \sup _{\tau \in\left[0,1-\tau_{0}\right]} \frac{\int_{\tau}^{1} \bar{K}_{c, \tau_{1}, \tau_{2}}^{*}(r) d K_{c, \tau_{1}, \tau_{2}}^{*}(r)}{\sqrt{\pi_{c, \tau_{1}, \tau_{2}}(1) \int_{\tau}^{1} \bar{K}_{c, \tau_{1}, \tau_{2}}^{*}(r)^{2} d r}} \equiv L_{c, \tau_{1}, \tau_{2}}^{* H B}
\end{aligned}
$$

where

$$
\begin{aligned}
& \tilde{K}_{c, \tau_{1}, \tau_{2}}^{*}(r)=K_{c, \tau_{1}, \tau_{2}}^{*}(r)-\frac{1}{r} \int_{0}^{r} K_{c, \tau_{1}, \tau_{2}}^{*}(s) d s \\
& \bar{K}_{c, \tau_{1}, \tau_{2}}^{*}(r)=K_{c, \tau_{1}, \tau_{2}}^{*}(r)-\int_{0}^{1} K_{c, \tau_{1}, \tau_{2}}^{*}(s) d s
\end{aligned}
$$

with

$$
K_{c, \tau_{1}, \tau_{2}}^{*}(r)= \begin{cases}W(r) & r \leq \tau_{1} \\ e^{\left(r-\tau_{1}\right) c} W\left(\tau_{1}\right)+\int_{\tau_{1}}^{r} e^{(r-s) c} d W(s) & \tau_{1}<r \leq \tau_{2} \\ W\left(\tau_{1}\right)+W(r)-W\left(\tau_{2}\right) & r>\tau_{2}\end{cases}
$$

and

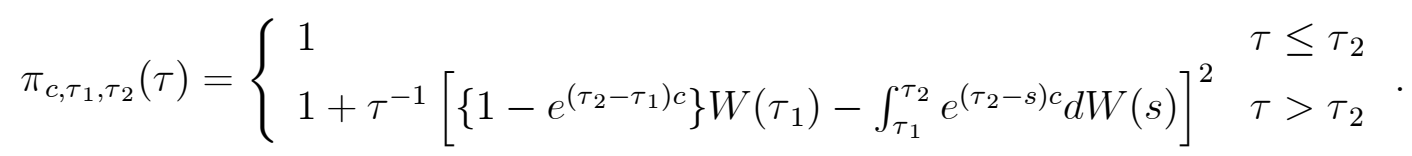

Remark 1 The limit distributions of $P W Y$ and $H B$ under the null hypothesis $H_{0}: \delta=$ 0 are given by $L_{0, \tau_{1}, \tau_{2}}^{P W Y}$ and $L_{0, \tau_{1}, \tau_{2}}^{H B}$, respectively, i.e. the limits obtained from Theorem 1(i) with $c$ set to zero. These null limits continue to hold for serially correlated $v_{t}$, provided the usual lagged difference augmentation is applied to the regressions (1) and $(2)$.

Asymptotic null critical values for $\tau_{0}=0.1$ (as used in PWY and HB) are reported in Table 1; these were generated by direct simulation of $L_{0, \tau_{1}, \tau_{2}}^{P W Y}$ and $L_{0, \tau_{1}, \tau_{2}}^{H B}$, approximating the Wiener processes in the limiting functionals using $\operatorname{IID} N(0,1)$ random variates, with the integrals approximated by normalized sums of 1,000 steps. $^{1}$ Here and throughout the paper, simulations were conducted using 50,000 Monte Carlo replications.

Figure 1 plots local asymptotic power curves of nominal 0.05-level $P W Y$ and $H B$ tests for $c \in\{0,0.5,1.0, \ldots, 24.0\}$, again obtained via direct simulation of the above limits. Figures 1(a) and 1(b) report results where the explosive regime lies in the middle of the sample and Case 1 holds, with $\left(\tau_{1}, \tau_{2}\right)=(0.45,0.55)$ in Figure 1 (a) and $\left(\tau_{1}, \tau_{2}\right)=(0.40,0.60)$ in Figure $1(\mathrm{~b})$, i.e. non-collapsing centrally located explosive periods of sample proportion duration 0.1 and 0.2 , respectively. Figures $1(\mathrm{c})$ and $1(\mathrm{~d})$

\footnotetext{
${ }^{1} \mathrm{HB}$ consider the case of no deterministic components in $y_{t}$, and the case of a non-zero mean and trend in $y_{t}$; here we allow for a non-zero mean only.
} 
report powers for the same settings as in Figures 1(a) and 1(b), respectively, except now for Case 2, such that the explosive period is collapsing. Figures 1(e) and 1(f) present powers when explosive periods of sample proportion duration 0.1 and 0.2 run up to the end of the sample, i.e. $\left(\tau_{1}, \tau_{2}\right)=(0.90,1.00)$ and $\left(\tau_{1}, \tau_{2}\right)=(0.80,1.00)$, respectively (note that in these cases the third regime in (3) is redundant).

Consider first Figures 1(a) and 1(b), where the explosive period does not collapse. We observe that local asymptotic power increases with the magnitude of the explosive deviation $c$, and also with the length of the explosive period. The rate of increase is much faster for $P W Y$ than for $H B$, with substantial power advantages offered by the former test relative to the latter, particularly in the case of the shorter explosive regime.

In Figures 1(c) and 1(d), where the explosive period is now subject to collapse, the power curves for $P W Y$ are seen to be largely unchanged from their non-collapsing counterparts. This feature arises since the supremum of the forward recursive statistics involved in $P W Y$ tends to occur when the associated sub-sample does not contain the post-explosive period of the data. In contrast, the collapsing explosive regime leads to a dramatic fall in the power levels associated with $H B$; indeed, power is less than size and approaches zero as $c$ (the locally explosive parameter) increases. This phenomenon is driven by the behaviour of the $\pi_{c, \tau_{1}, \tau_{2}}(1)$ term (which is the limit of $\hat{\sigma}_{H B}^{2} / \sigma^{2}$ ) in the denominator of the limit of $H B$. This term is a positive random variable for which the mean can be shown to be

$$
E\left\{\pi_{c, \tau_{1}, \tau_{2}}(1)\right\}=1+\tau_{1}\left(1-e^{\left(\tau_{2}-\tau_{1}\right) c}\right)^{2}+\frac{e^{2\left(\tau_{2}-\tau_{1}\right) c}-1}{2 c},
$$

which is an exponentially increasing function of $c$. Heuristically, as $c$ increases, this term leads to the value of $C_{\tau}$ approaching zero (across all $\tau$ ), and hence $H B$ similarly approaches zero. On an intuitive note, such behaviour is to be expected since, for all $C_{\tau}, \hat{\sigma}_{H B}^{2}$ is calculated using the full sample of residuals, and is therefore polluted by the impact of the collapse, whose magnitude is an increasing function of $\delta$. In contrast, for $P W Y$ we see that $\hat{\sigma}_{P W Y}^{2}$ in $D F_{\tau}$ is calculated using a subset of the data, which is unpolluted by the collapse for any $\tau \leq \tau_{2}$; consequently, the $P W Y$ supremum is typically obtained for a $D F_{\tau}$ statistic where $\tau \leq \tau_{2}$. Here, $\pi_{c, \tau_{1}, \tau_{2}}(\tau)$ (the limit of $\left.\hat{\sigma}_{P W Y}^{2} / \sigma^{2}\right)$ takes a value of unity irrespective of $c$, and so the behaviour of the error variance estimator does not influence the $P W Y$ test statistic in the way that is seen for $H B$.

Essentially, the inferior power of the $H B$ test relative to $P W Y$ seen in both Figures 1(a)-1(b) and 1(c)-1(d) arises since the backward recursive statistics involved in $H B$ are necessarily calculated over sample periods polluted by the post-explosive regime. Moreover, when this post-explosive regime involves reversion to a pre-explosive level of the series, the backward recursive statistics are more fundamentally impacted, to the extent that the resulting $H B$ test has trivial power across $c$. On the basis of Figures 1(a)-1(d), therefore, a clear preference for the $P W Y$ test results. In Figures 1(e) and $1(\mathrm{f})$, where the explosive periods run up to the end of the sample, we find that the power ranking of $P W Y$ and $H B$ is reversed, with $H B$ offering reasonable levels of power gain over $P W Y$. Here, the backward recursive statistics involved in $H B$ are no longer 
polluted by a post-explosive regime, and the supremum of the statistics now tends to occur when the associated sub-sample contains the entirety of the explosive regime.

It is noteworthy that $P W Y$ maintains decent levels of power across the different explosive settings considered, a property that is not displayed by $H B$; as such, it is clearly the more reliable of the two procedures. However, it is also apparent that while $P W Y$ dominates $H B$ in the majority of cases, when the series under test contains an explosive period that is still operative at the sample end, it is $H B$ that becomes the preferred test. This gives rise to the question of whether a composite procedure can be constructed that capitalizes on the relative advantages of both the individual tests; this is pursued further in section 5 below.

\section{Finite sample power comparison}

To assess the extent to which these local asymptotic power comparisons are accurate predictors of finite sample behaviour, we also consider a number of finite sample simulations. Figures 2 and 3 plot finite sample power curves of nominal 0.05-level $P W Y$ and $H B$ tests for $T=300$ and $T=600$ for $\delta \in\{0,0.001,0.002, \ldots, 0.080\}$ and $\delta \in\{0,0.001,0.002, \ldots, 0.040\}$, respectively (note that $\delta=0.08$ and $\delta=0.04$ correspond to $\delta=1+c / T$ with $c=24$ when $T=300$ and $T=600$, respectively, so that the range of $\delta$ values matches those used in the local asymptotic power simulations). Here, $v_{t} \sim \operatorname{IID} N(0,1)$ and the settings used for $\tau_{1}$ and $\tau_{2}$ mirror those of Figure 1 , with both Case $1\left(y^{*}=0\right)$ and Case $2\left(y^{*}=y_{\left\lfloor\tau_{1} T\right\rfloor}-y_{\left\lfloor\tau_{2} T\right\rfloor}\right)$ again considered. We see that in each case of Figures 2 and 3, the finite sample powers generally align closely with their local asymptotic counterparts, particularly in the case of the larger sample size $T=600$, thus the same comments made above regarding the relative powers of the tests apply equally in finite samples.

In order to assess any possible effects of non-normality in finite samples, Figure 4 reports finite sample power curves for $T=300$ for the same DGPs as in Figure 2, but with $v_{t} \sim I I D t_{5}$. The results are found to be almost identical to those obtained under normality of the innovations.

To further explore the effect that the location of the explosive period, relative to the end of the sample, has on the performance of the test procedures, we undertake the following exercise. We set $v_{t} \sim \operatorname{IID} N(0,1), T=300, \delta=1.05$ and $\left(\tau_{1}, \tau_{2}\right)=$ $(0.70,0.80)$ so that an explosive period of length $0.1 T$ occurs relatively late in the sample. We then examine the performance of the tests with the end date of the sample (denoted $E$ ) varied from $E=\left\lfloor\tau_{1} T\right\rfloor=210$ to $E=300$, with the $P W Y$ and $H B$ tests applied to a varying effective sample size, $T^{*}$, ranging from $T^{*}=210$ (i.e. observations $t=1, \ldots, 210)$ to $T^{*}=T=300$ (i.e. observations $t=1, \ldots, 300$ ). When $E=210$, no explosive period is contained in the sample, then as $E$ increases, the explosive period following the initial unit root regime is increasing in length, up to the point $E=240$, where the series contains the largest possible explosive period with no final unit root regime. As $E$ increases still further, the final unit root period increases in length, up to the point $E=300$, where the full sample is recovered. Figure 5 reports results for such simulations, where each point on the power curve represents the outcome of a 
simulation for a particular value of $E$; Figures 5(a) and 5(b) correspond to Case 1 and Case 2, respectively.

Figure 5(a), the case where the explosive regime does not collapse, shows the power of both $P W Y$ and $H B$ rising in $E$ as the length of the explosive period increases, up to $E=240$, which represents the end of the explosive period. For this range of $E$, all series contain an explosive regime that runs to the sample's end, and, in line with the results of Figures 1(e) and 1(f), $H B$ is clearly the more powerful test. For larger values of $E$, where the sample now includes a post-explosive unit root regime of increasing length, the power of $P W Y$ stays pretty much constant, while the power of $H B$ starts to decline steadily (becoming lower than that of $P W Y$ for, roughly, $E>260$ ). In Figure 5(b), where the explosive regime collapses to pre-explosive levels, the behaviour of the two tests across $E$ up to $E=240$ is, of course, identical to that in Figure 5(a). Thereafter, however, while $P W Y$ again retains pretty much constant power for larger values of $E$, the power of $H B$ falls immediately to zero, and remains there. It is noteworthy that inclusion of as little as one observation of the collapsed regime induces this behaviour.

Figure 6 reports results for a similar simulation exercise, where $T=300$ and $\delta=$ 1.05 as before, but now $\left(\tau_{1}, \tau_{2}\right)=(0.20,0.30)$, so that the explosive period occurs relatively early. Here we examine the performance of the procedures with the start date of the sample (denoted $S$ ) varied from $S=1$ to $S=\left\lfloor\tau_{2} T+1\right\rfloor=91$, so that the $P W Y$ and $H B$ tests are again applied to varying effective sample sizes, ranging from $T^{*}=300$ (i.e. observations $t=1, \ldots, 300$ ) to $T^{*}=210$ (i.e. observations $t=91, \ldots, 300$ ). As $S$ increases, the initial unit root period prior to the explosive regime is decreasing in length, up to the point $S=\left\lfloor\tau_{1} T+1\right\rfloor=61$, where no initial unit root period is present and the series begins explosively. As $S$ increases further, the explosive period now decreases in length, up to the point $S=\left\lfloor\tau_{2} T+1\right\rfloor=91$, where no explosive period is now present. As before, Figures 6(a) and 6(b) correspond, respectively, to Case 1 and Case 2.

In both Figures 6(a) and 6(b) we observe that $H B$ only ever has trivial levels of power across $S$. This arises because there is always a post-explosive unit root regime of long duration (210 observations) present in the sample irrespective of the value of $S$. As regards $P W Y$, it has decent, and roughly constant, power for up to about $S=50$, after which point the reduced sample size negatively affects power, particularly for $S>61$ where less observations of the explosive period are included in the sample. For $S \geq 69$, the minimum sample length constraint on the set of $D F_{\tau}$ statistics over which the supremum is taken results in all such statistics being calculated over a subsample that includes observations from the post-explosive regime; this has the effect of reducing test power to trivial levels.

We can therefore conclude that, in general terms, $P W Y$ is better suited to detecting explosive regimes that occur early or towards the middle of the sample, while $H B$ is better when these explosive regimes occur towards the end of the sample, provided the associated explosive regime does not collapse prior to the sample's end. 


\section{$5 \quad$ A union of rejections strategy}

In practice we cannot assume knowledge of where in a given sample an explosive regime occurs (should one occur at all); nor can we assume whether or not such a regime is associated with a collapse. In such situations it is therefore unclear as to which of $P W Y$ and $H B$ will have the greater potential to detect explosive behaviour. One strategy would be to simply apply $P W Y$ alone since post-explosive unit root regimes do not have the potential to dramatically lower its power, unlike $H B$. Such an approach would, of course, mean we sacrifice the power advantage offered by $H B$ when post-explosive unit root regimes do not cause low power issues (for example, when the explosive period runs to the sample end-point).

We now consider the possibility of harnessing the desirable properties of both tests using a hybrid procedure based on $P W Y$ and $H B$. The approach we adopt here is the following simple union of rejections decision rule:

$$
U R \text { : Reject } H_{0} \text { if } P W Y>\lambda_{\gamma} c v_{\gamma}^{P W Y} \text { or } H B>\lambda_{\gamma} c v_{\gamma}^{H B}
$$

where $c v_{\gamma}^{P W Y}$ and $c v_{\gamma}^{H B}$ denote the asymptotic null critical values of $P W Y$ and $H B$ for a significance level $\gamma$ (see Table 1). Here $\lambda_{\gamma}$ is a scaling constant calculated such that the asymptotic size associated with $U R$ is equal to the nominal size $\gamma$. The decision rule $U R$ can also be written as

$$
\text { Reject } H_{0} \text { if } \max \left(P W Y, \frac{c v_{\gamma}^{P W Y}}{c v_{\gamma}^{H B}} H B\right)>\lambda_{\gamma} c v_{\gamma}^{P W Y}
$$

where, for $\delta=0$,

$$
\max \left(P W Y, \frac{c v_{\gamma}^{P W Y}}{c v_{\gamma}^{H B}} H B\right) \stackrel{d}{\rightarrow} \max \left(L_{0, \tau_{1}, \tau_{2}}^{P W Y}, \frac{c v_{\gamma}^{P W Y}}{c v_{\gamma}^{H B}} L_{0, \tau_{1}, \tau_{2}}^{H B}\right)
$$

and for $\delta=1+c / T, c>0$,

$$
\max \left(P W Y, \frac{c v_{\gamma}^{P W Y}}{c v_{\gamma}^{H B}} H B\right) \stackrel{d}{\rightarrow}\left\{\begin{array}{ll}
\max \left(\begin{array}{l}
L_{c, \tau_{1}, \tau_{2}}^{P W Y}, \frac{c v_{\gamma}^{P W Y}}{c v_{\gamma}^{H B}} L_{c, \tau_{1}, \tau_{2}}^{H B} \\
\max \left(L_{c, \tau_{1}, \tau_{2}}^{* P W Y}, \frac{c v_{\gamma}^{P W Y}}{c v_{\gamma}^{H B}} L_{c, \tau_{1}, \tau_{2}}^{* H B}\right.
\end{array}\right) \text { Case } 1 \\
\text { Case 2 }
\end{array} .\right.
$$

At a given significance level $\gamma$, the appropriate value for the constant $\lambda_{\gamma}$ is obtained by simulating the limit distribution of the right-hand-side of (5), calculating the $\gamma$ level critical value for this empirical distribution, say $c v_{\gamma}^{U}$, and then computing $\lambda_{\gamma}=$ $c v_{\gamma}^{U} / c v_{\gamma}^{P W Y}$. These values are given in Table 1 for the conventional significance levels.

Figures 1-6 also show the powers of $U R$ at the nominal 0.05-level. Figure 1 shows that across values of $c$, the local asymptotic power of $U R$ always tracks very close to (that is, just below) whichever of $P W Y$ or $H B$ possesses the higher level of power. In this sense, $U R$ forms a near-envelope of the available power from $P W Y$ and $H B$, displaying almost all the gains offered by $P W Y$ when the explosive regime ends within the sample, while simultaneously capturing the $H B$ power advantages for explosive regimes that are still active at the end of the sample period. The same patterns are observed in finite samples, with Figures 2, 3 and 4 showing a very close correspondence 
to the results in Figure 1. In Figures 5 and 6 , the benefits of $U R$ can again be clearly seen across sample end values, $E$, and sample start values, $S$, with $U R$ always closely tracking the power curve of the better performing test across each segment. As such, $U R$ would seem to be a highly effective means of combining inference from $P W Y$ and $H B$, offering a size controlled and robust method for the detection of explosive behaviour in a time series.

\section{Empirical illustration}

In this section we illustrate the differing behaviour of the $P W Y$ and $H B$ tests, along with the $U R$ strategy, by applying the procedures to monthly data on the Nasdaq composite price index, as used by PWY (and also reconsidered by HB). We employ data over the same period as PWY, i.e. 1973:2-2005:6 $(T=389)$, using the composite price index adjusted for dividends, and also the composite dividend series derived from the the dividend yields; the data were obtained from Datastream. Logarithms of the real values of the prices and dividends are used, with the nominal data converted using (seasonally adjusted) US consumer price index data obtained from the Federal Reserve Bank of St. Louis. The series are plotted in Figure 7.

In order to assess whether the inference of the tests is sensitive to the end point of the sample (in the manner of the power results reported in Figure 5) we applied the procedures to sample periods from 1973:2 to varying end points from $E=1996: 1$ to $E=2005: 6$, this last end date representing the full PWY sample period. When applying the $P W Y$ and $H B$ tests, the underlying DF statistics were computed from the OLS regressions (1) and (2) augmented with a number of lagged difference terms, the number being selected according to the Bayes information criterion, with a maximum of 12 , reflecting the monthly nature of the data. Figure 8 reports the results from this application, plotting the $P W Y$ and $H B$ statistics across $E$. In the figures, a straight line is plotted at the level of the asymptotic 0.05 -level critical value for $P W Y$ (i.e. 1.411, see Table 1), and in order to allow comparison with a common critical value, the reported $H B$ test statistics are multiplied by the ratio of the $P W Y$ and $H B$ asymptotic 0.05 -level critical values (i.e. $1.411 / 1.608=0.877$ ). Figure 8 also reports results for the union of rejections strategy $U R$. In order to again allow comparison with a common critical value, the reported values are $\max \{P W Y, 0.877 H B\} / 1.171$, based on (6) with the critical values and $\lambda_{\gamma}$ value from Table 1 .

For the price series, we see that $P W Y$ rejects the unit root null in favour of explosive behaviour at the 0.05 -level for all samples with end points at $E=1999: 1$ or later. In contrast, $H B$ finds evidence of explosiveness when the sample ends at dates $E=2000: 12$ and earlier (and also when $E=2001: 2$ ), but does not reject the null for any later end dates. Hence it is only for samples with end dates in the range $E=1999: 1$ to $E$ $=2000: 12$ (and $E=2001: 2$ ) where both tests reject; outside of this range, inference depends on the particular testing approach adopted. For $U R$, however, we find that rejections are obtained for all samples with end points $E=1998: 11$ onwards and the vast majority of the end dates prior to this point, delivering robust inference across a much wider range of sample end dates than available from either constituent test. 
Indeed, the $U R$ procedure is close to capturing all the rejections of both $P W Y$ and $H B$, with 104 end points out of the 114 considered resulting in a rejection by $P W Y$ and/or $H B$ being matched with a rejection by $U R$. As might be expected, no evidence against the unit root null is found by any of the procedures for the dividend series, suggesting that an explosive period in the price series can be interpreted as a bubble.

Visual inspection of the Nasdaq price series plotted in Figure 7 might suggest the presence of explosive bubble behaviour up to the maximum value observed in 2000:3, followed by a collapse thereafter. The pattern of test rejections we obtain are consistent with such a notion, given our earlier simulation results. Specifically, samples which include a number of post-collapse observations cause $H B$ to fail to detect evidence of a bubble, while these observations have no effect on $P W Y$. For samples where the putative bubble is still in evidence at the end of the period considered, i.e. pre-2000:3, $H B$ is more likely to detect explosive behaviour than $P W Y$.

From a practitioner's perspective, applying the tests with the benefit of the full sample data spanning 1973:2-2005:6 yields a rejection in favour of bubble behaviour only with $P W Y$. If the tests were applied in 2000:3 (or a number of months either side) then both $P W Y$ and $H B$ would have rejected. However, if one were to have implemented the tests prior to 1999 (with the sample therefore ending at such a time), $P W Y$ would have failed to find evidence of a bubble, and a rejection would only have been obtained with $H B$. This inconsistency of inference associated with $P W Y$ and $H B$ is naturally a cause of concern, yet is almost completely eradicated by use of the $U R$ strategy, which would have consistently indicated evidence of a bubble over this period for almost all points in time at which the application was conducted.

\section{Conclusion}

Recent research on testing for the presence of explosive rational asset price bubbles has focused on the use of tests derived from conventional linear autoregressive models that are estimated recursively to detect explosive autoregressive regimes in an otherwise unit root process. When applied to an asset price series and the associated fundamentals series, tests of this type proposed by PWY and HB offer methods of detecting explosive rational bubbles. This paper has focused on the relative local asymptotic and finite sample power of the forward recursive PWY and backward recursive HB tests when the series contains a single explosive period, possibly with some form of collapse. Simulations of both asymptotic and finite sample behaviour showed that the power of these two tests can differ quite dramatically depending on the location of the explosive regime, and whether such a regime ends in collapse; overall, we find that the PWY test is preferred for detecting explosive regimes that occur early or towards the middle of the sample, while the HB test is preferred when the explosive behaviour occurs near the end of the sample, provided the explosive regime does not end in collapse. Motivated by this pattern of relative test power, we proposed a simple union of rejections strategy that, for a given specification of the explosive regime, attains power close to the better of the individual PWY and HB tests. Since the timing, duration and collapse properties of a putative bubble period are typically unknown to the practitioner, so 
that in practice it is not known which of PWY or HB would offer the greater ability to detect an explosive bubble, our proposed composite approach offers a more robust approach to the testing problem, as further evidenced by our empirical illustration, and we envisage it being useful to applied researchers.

While the focus of our analysis has addressed procedures for detecting the presence of an explosive regime, it is also possible to use the statistics underlying $P W Y$ and $H B$ to date the beginning and end of such explosive behaviour. PWY suggest timestamping the origination and termination of the explosive regime based on the dates for which the $D F_{\tau}$ statistics exceed (diverging) critical values, and a corresponding approach could be developed using the $C_{\tau}$ statistics of HB. An alternative approach to dating the start of an explosive regime is proposed by HB, whereby the argmax of the backward recursive $C_{\tau}$ statistics is employed as a regime-change date estimator, and, once again, a corresponding approach could be devised based on taking the argmax of the forward recursive $D F_{\tau}$ statistics. We leave a proper comparison of such dating schemes, and potential combinations thereof, as an avenue for future research.

\section{References}

Diba, B.T. and Grossman, H.I. (1988). Explosive rational bubbles in stock prices? American Economic Review, 78, 520-530.

Evans, G.W. (1991). Pitfalls in testing for explosive bubbles in stock prices. American Economic Review, 81, 922-930.

Hall, S., Psaradakis, Z. and Sola, M. (1999). Detecting periodically collapsing bubbles: a Markov-switching unit root test. Journal of Applied Econometrics, 14, 143-154.

Harvey, D.I., Leybourne, S.J. and Taylor, A.M.R. (2009). Unit root testing in practice: dealing with uncertainty over the trend and initial condition (with commentaries and rejoinder). Econometric Theory, 25, 587-667.

Harvey, D.I., Leybourne, S.J. and Taylor, A.M.R. (2012). Testing for unit roots in the presence of uncertainty over both the trend and initial condition. Journal of Econometrics, 169, 188-195.

Homm, U. and Breitung, J. (2012). Testing for speculative bubbles in stock markets: a comparison of alternative methods. Journal of Financial Econometrics, 10, 198-231.

Phillips, P.C.B. (1987). Towards a unified asymptotic theory for autoregression. Biometrika, 74, 535-547.

Phillips, P.C.B., Wu, Y. and Yu, J. (2011). Explosive behaviour in the 1990s Nasdaq: when did exuberance escalate stock values? International Economic Review, 52, 201-226. 


\section{Appendix: Proof of Theorem 1}

(i) By backward substitution in (3) we obtain

$$
y_{t}= \begin{cases}\sum_{i=1}^{t} v_{i} & t=2, \ldots,\left\lfloor\tau_{1} T\right\rfloor \\ (1+\delta)^{t-\left\lfloor\tau_{1} T\right\rfloor} \sum_{i=1}^{\left\lfloor\tau_{1} T\right\rfloor} v_{i}+\sum_{i=\left\lfloor\tau_{1} T+1\right\rfloor}^{t}(1+\delta)^{t-i} v_{i} & t=\left\lfloor\tau_{1} T+1\right\rfloor, \ldots,\left\lfloor\tau_{2} T\right\rfloor \\ (1+\delta)^{\left\lfloor\tau_{2} T\right\rfloor-\left\lfloor\tau_{1} T\right\rfloor} \sum_{i=1}^{\left\lfloor\tau_{1} T\right\rfloor} v_{i} & t=\left\lfloor\tau_{2} T+1\right\rfloor, \ldots, T \\ \quad+\sum_{i=\left\lfloor\tau_{1} T+1\right\rfloor}^{\left\lfloor\tau_{2} T\right\rfloor}(1+\delta)^{t-i} v_{i}+\sum_{i=\left\lfloor\tau_{2} T+1\right\rfloor}^{t} v_{i} & \end{cases}
$$

and subsequently

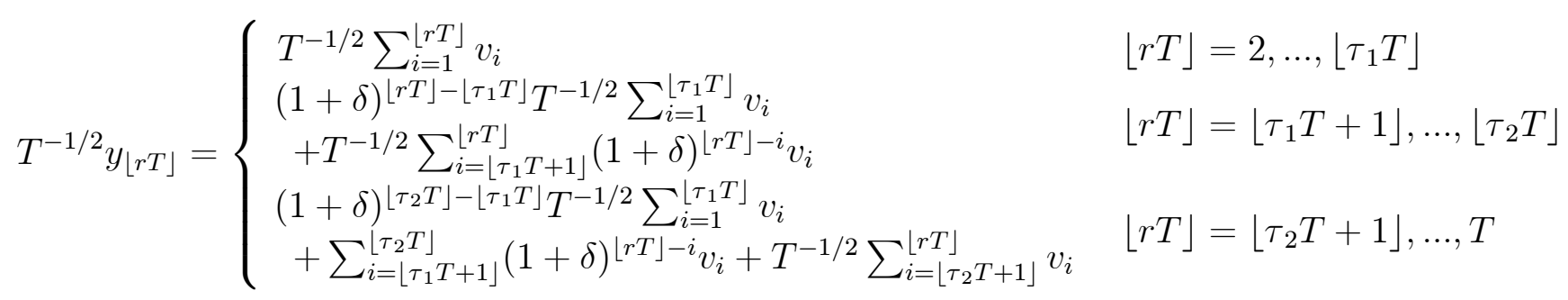

Under $\delta=c / T$, for $0<a<b<1,(1+\delta)^{\lfloor b T\rfloor-\lfloor a T\rfloor}=e^{(b-a) c}+o(1)$, and then, following Phillips (1987) we find

$$
\begin{aligned}
\sigma^{-1} T^{-1 / 2} y_{\lfloor r T\rfloor} & \stackrel{d}{\rightarrow} \begin{cases}W(r) & r \leq \tau_{1} \\
e^{\left(r-\tau_{1}\right) c} W\left(\tau_{1}\right)+\int_{\tau_{1}}^{r} e^{(r-s) c} d W(s) & \tau_{1}<r \leq \tau_{2} \\
e^{\left(\tau_{2}-\tau_{1}\right) c} W\left(\tau_{1}\right)+\int_{\tau_{1}}^{\tau_{2}} e^{\left(\tau_{2}-s\right) c} d W(s)+W(r)-W\left(\tau_{2}\right) & r>\tau_{2}\end{cases} \\
& =K_{c, \tau_{1}, \tau_{2}}(r) .
\end{aligned}
$$

It is straightforward to show that $\hat{\sigma}_{P W Y}^{2}=\lfloor\tau T\rfloor^{-1} \sum_{t=1}^{\lfloor\tau T\rfloor} \Delta y_{t}^{2}+o_{p}(1)$ and $\hat{\sigma}_{H B}^{2}=$ $T^{-1} \sum_{t=1}^{T} \Delta y_{t}^{2}+o_{p}(1)$. Then, since we can also show that $\lfloor\tau T\rfloor^{-1} \sum_{t=1}^{\lfloor\tau T\rfloor} \Delta y_{t}^{2} \stackrel{p}{\rightarrow} \sigma^{2}$ for any $\tau$, we find that $\hat{\sigma}_{P W Y}^{2} \stackrel{p}{\rightarrow} \sigma^{2}$ and $\hat{\sigma}_{H B}^{2} \stackrel{p}{\rightarrow} \sigma^{2}$. The stated limits for $P W Y$ and $H B$ then follow from an application of the continuous mapping theorem.

(ii) The limits $K_{c, \tau_{1}, \tau_{2}}^{*}(r)$ and $K_{c, \tau_{1}, \tau_{2}}(r)$ are identical apart from when $r>\tau_{2}$. In this case, the third partition of (7) is replaced by

$$
y_{t}=\sum_{i=1}^{\left\lfloor\tau_{1} T\right\rfloor} v_{i}+\sum_{i=\left\lfloor\tau_{2} T+1\right\rfloor}^{\lfloor r T\rfloor} v_{i}, \quad t=\left\lfloor\tau_{2} T+1\right\rfloor, \ldots, T
$$

and then

$$
\sigma^{-1} T^{-1 / 2} y_{\lfloor r T\rfloor} \stackrel{d}{\rightarrow} W\left(\tau_{1}\right)+W(r)-W\left(\tau_{2}\right), \quad r>\tau_{2}
$$


Using (7) and (8),

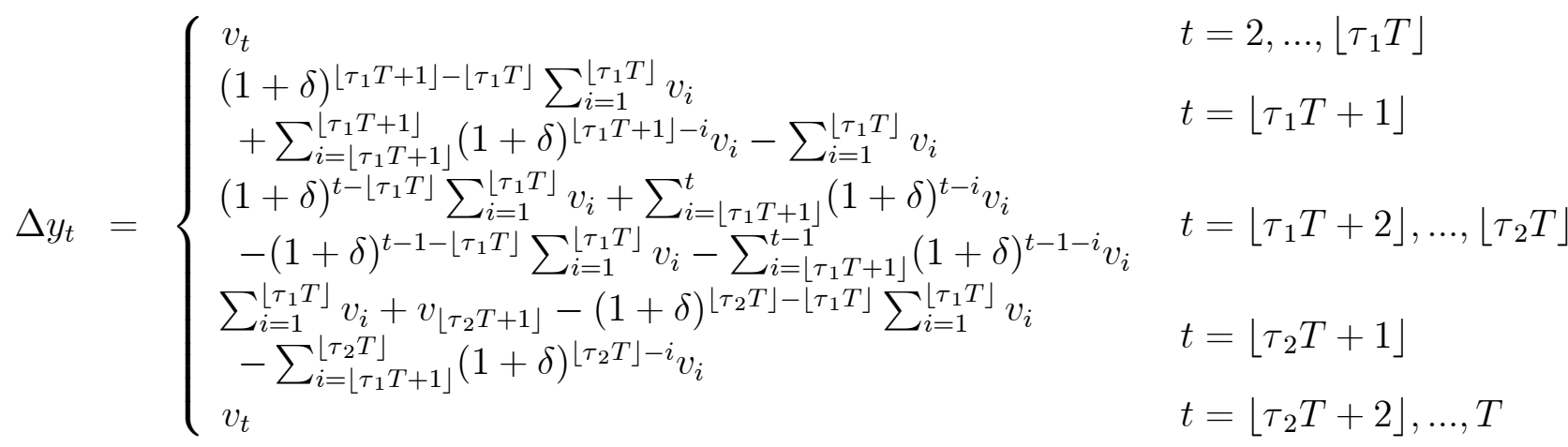

$$
\begin{aligned}
& = \begin{cases}v_{t} & t=2, \ldots,\left\lfloor\tau_{1} T\right\rfloor \\
\delta \sum_{i=1}^{\left\lfloor\tau_{1} T\right\rfloor} v_{i}+(1+\delta) v_{\left\lfloor\tau_{1} T+1\right\rfloor} & t=\left\lfloor\tau_{1} T+1\right\rfloor \\
v_{t}+\delta \sum_{i=\left\lfloor\tau_{1} T+1\right\rfloor}^{t-1}(1+\delta)^{t-1-i} v_{i} & t=\left\lfloor\tau_{1} T+2\right\rfloor, \ldots,\left\lfloor\tau_{2} T\right\rfloor \\
\quad+\delta(1+\delta)^{t-\left\lfloor\tau_{1} T\right\rfloor-1} \sum_{i=1}^{\left\lfloor\tau_{1} T\right\rfloor} v_{i} & \\
v_{\left\lfloor\tau_{2} T+1\right\rfloor}+\left\{1-(1+\delta)^{\left\lfloor\tau_{2} T\right\rfloor-\left\lfloor\tau_{1} T\right\rfloor}\right\} \sum_{i=1}^{\left\lfloor\tau_{1} T\right\rfloor} v_{i} & t=\left\lfloor\tau_{2} T+1\right\rfloor \\
\quad-\sum_{i=\left\lfloor\tau_{1} T+1\right\rfloor}^{\left\lfloor\tau_{2} T\right\rfloor}(1+\delta)^{\left\lfloor\tau_{2} T\right\rfloor-i} v_{i} & t=\left\lfloor\tau_{2} T+2\right\rfloor, \ldots, T \\
v_{t} & t\end{cases}
\end{aligned}
$$

Since $\delta=c / T$ we then find that

$$
\Delta y_{t}= \begin{cases}v_{t} & t=2, \ldots,\left\lfloor\tau_{1} T\right\rfloor \\ v_{\left\lfloor\tau_{1} T+1\right\rfloor}+o_{p}(1) & t=\left\lfloor\tau_{1} T+1\right\rfloor \\ v_{t}+o_{p}(1) & t=\left\lfloor\tau_{1} T+2\right\rfloor, \ldots,\left\lfloor\tau_{2} T\right\rfloor \\ \left\{1-(1+c / T)^{\left\lfloor\tau_{2} T\right\rfloor-\left\lfloor\tau_{1} T\right\rfloor}\right\} \sum_{i=1}^{\left\lfloor\tau_{1} T\right\rfloor} v_{i} & t=\left\lfloor\tau_{2} T+1\right\rfloor \\ -\sum_{i=\left\lfloor\tau_{1} T+1\right\rfloor}^{\left\lfloor\tau_{2} T\right\rfloor}(1+c / T)^{\left\lfloor\tau_{2} T\right\rfloor-i} v_{i}+o_{p}\left(T^{-1 / 2}\right) & t=\left\lfloor\tau_{2} T+2\right\rfloor, \ldots, T \\ v_{t} & t \tau^{2} T\end{cases}
$$

As regards $\hat{\sigma}_{P W Y}^{2}$, we can again show that $\hat{\sigma}_{P W Y}^{2}=\lfloor\tau T\rfloor^{-1} \sum_{t=1}^{\lfloor\tau T\rfloor} \Delta y_{t}^{2}+o_{p}(1)$. Then for $\lfloor\tau T\rfloor \leq\left\lfloor\tau_{2} T\right\rfloor$, we find that $\lfloor\tau T\rfloor^{-1} \sum_{t=1}^{\lfloor\tau T\rfloor} \Delta y_{t}^{2}=\lfloor\tau T\rfloor^{-1} \sum_{t=1}^{\lfloor\tau T\rfloor} v_{t}^{2}+o_{p}(1) \stackrel{p}{\rightarrow} \sigma^{2}$. For $\lfloor\tau T\rfloor>\left\lfloor\tau_{2} T\right\rfloor$, write

$$
\lfloor\tau T\rfloor^{-1} \sum_{t=1}^{\lfloor\tau T\rfloor} \Delta y_{t}^{2}=\lfloor\tau T\rfloor^{-1} \sum_{t=1}^{\left\lfloor\tau_{2} T\right\rfloor} \Delta y_{t}^{2}+\lfloor\tau T\rfloor^{-1} \sum_{t=\left\lfloor\tau_{2} T\right\rfloor+1}^{\lfloor\tau T\rfloor} \Delta y_{t}^{2} .
$$

Here

$$
\begin{aligned}
\lfloor\tau T\rfloor^{-1} \sum_{t=1}^{\left\lfloor\tau_{2} T\right\rfloor} \Delta y_{t}^{2} & =\lfloor\tau T\rfloor^{-1}\left\lfloor\tau_{2} T\right\rfloor\left\lfloor\tau_{2} T\right\rfloor^{-1} \sum_{t=1}^{\left\lfloor\tau_{2} T\right\rfloor} v_{t}^{2}+o_{p}(1) \\
& \stackrel{p}{\rightarrow} \frac{\tau_{2}}{\tau} \sigma^{2} .
\end{aligned}
$$

Also,

$$
\lfloor\tau T\rfloor^{-1} \sum_{t=\left\lfloor\tau_{2} T\right\rfloor+1}^{\lfloor\tau T\rfloor} \Delta y_{t}^{2}=\lfloor\tau T\rfloor^{-1} \Delta y_{\left\lfloor\tau_{2} T\right\rfloor+1}^{2}+\lfloor\tau T\rfloor^{-1} \sum_{t=\left\lfloor\tau_{2} T\right\rfloor+2}^{\lfloor\tau T\rfloor} \Delta y_{t}^{2}
$$

where

$$
\begin{aligned}
\lfloor\tau T\rfloor^{-1} \sum_{t=\left\lfloor\tau_{2} T\right\rfloor+2}^{\lfloor\tau T\rfloor} \Delta y_{t}^{2} & =\lfloor\tau T\rfloor^{-1} \sum_{t=\left\lfloor\tau_{2} T\right\rfloor+2}^{\lfloor\tau T\rfloor} v_{t}^{2} \\
& =\lfloor\tau T\rfloor^{-1}\left(\lfloor\tau T\rfloor-\left\lfloor\tau_{2} T\right\rfloor\right)\left(\lfloor\tau T\rfloor-\left\lfloor\tau_{2} T\right\rfloor\right)^{-1} \sum_{t=\left\lfloor\tau_{2} T\right\rfloor+2}^{\lfloor\tau T\rfloor} v_{t}^{2} \\
& \stackrel{p}{\rightarrow} \frac{\tau-\tau_{2}}{\tau} \sigma^{2}
\end{aligned}
$$


while

$$
\begin{aligned}
\lfloor\tau T\rfloor^{-1} \Delta y_{\left\lfloor\tau_{2} T\right\rfloor+1}^{2}=\tau^{-1}[ & \left\{1-(1+c / T)^{\left\lfloor\tau_{2} T\right\rfloor-\left\lfloor\tau_{1} T\right\rfloor}\right\} T^{-1 / 2} \sum_{i=1}^{\left\lfloor\tau_{1} T\right\rfloor} v_{i} \\
& \left.-T^{-1 / 2} \sum_{i=\left\lfloor\tau_{1} T+1\right\rfloor}^{\left\lfloor\tau_{2} T\right\rfloor}(1+\delta)^{\left\lfloor\tau_{2} T\right\rfloor-i} v_{i}\right]^{2} \\
\stackrel{d}{\rightarrow} & \sigma^{2} \tau^{-1}\left[\left\{1-e^{\left(\tau_{2}-\tau_{1}\right) c}\right\} W\left(\tau_{1}\right)-\int_{\tau_{1}}^{\tau_{2}} e^{\left(\tau_{2}-s\right) c} d W(s)\right]^{2} .
\end{aligned}
$$

So,

$$
\lfloor\tau T\rfloor^{-1} \sum_{t=1}^{\lfloor\tau T\rfloor} \Delta y_{t}^{2} \stackrel{d}{\rightarrow} \sigma^{2}+\sigma^{2} \tau^{-1}\left[\left\{1-e^{\left(\tau_{2}-\tau_{1}\right) c}\right\} W\left(\tau_{1}\right)-\int_{\tau_{1}}^{\tau_{2}} e^{\left(\tau_{2}-s\right) c} d W(s)\right]^{2} .
$$

Taking the limits together, we see that

$$
\begin{aligned}
& \hat{\sigma}_{P W Y}^{2} \stackrel{d}{\rightarrow} \sigma^{2} \begin{cases}1 & \tau \leq \tau_{2} \\
1+\tau^{-1}\left[\left\{1-e^{\left(\tau_{2}-\tau_{1}\right) c}\right\} W\left(\tau_{1}\right)-\int_{\tau_{1}}^{\tau_{2}} e^{\left(\tau_{2}-s\right) c} d W(s)\right]^{2} & \begin{array}{l}
\tau>\tau_{2}
\end{array}\end{cases} \\
& =\sigma^{2} \pi_{c, \tau_{1}, \tau_{2}}(\tau) \text {. }
\end{aligned}
$$

Finally, for $\hat{\sigma}_{H B}^{2}$, we can show that $\hat{\sigma}_{H B}^{2}=T^{-1} \sum_{t=1}^{T} \Delta y_{t}^{2}+o_{p}(1)$. It follows that its limit is the same as that of $\hat{\sigma}_{P W Y}^{2}$ upon setting $\tau=1$, i.e. $\sigma^{2} \pi_{c, \tau_{1}, \tau_{2}}(1)$. 
Table 1. Asymptotic critical values for $P W Y$ and $H B$ at the $\gamma$ significance level, and $\lambda_{\gamma}$ values for $U R$

\begin{tabular}{cccc}
\hline & \multicolumn{2}{c}{ Critical values } & \\
\cline { 2 - 3 }$\gamma$ & $P W Y$ & $H B$ & $\lambda_{\gamma}$ values \\
\hline 0.10 & 1.138 & 1.245 & 1.240 \\
0.05 & 1.411 & 1.608 & 1.171 \\
0.01 & 1.929 & 2.259 & 1.104 \\
\hline
\end{tabular}




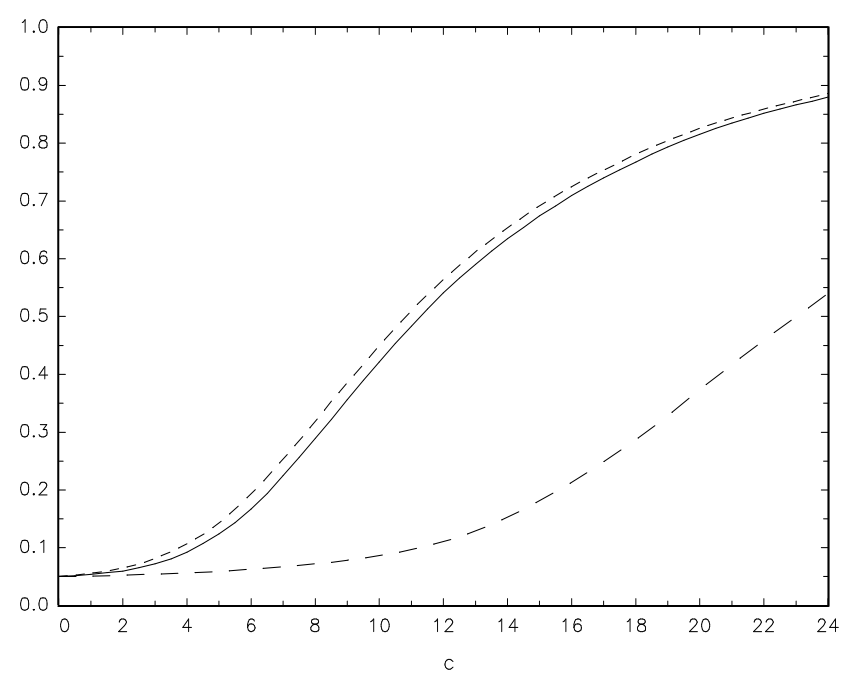

(a) $\left(\tau_{1}, \tau_{2}\right)=(0.45,0.55)$, Case 1

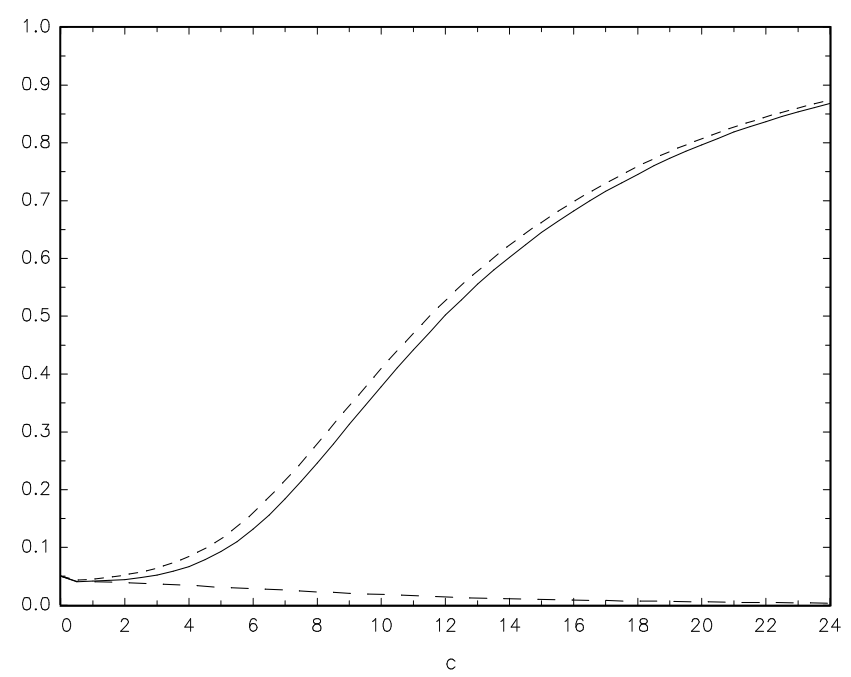

(c) $\left(\tau_{1}, \tau_{2}\right)=(0.45,0.55)$, Case 2

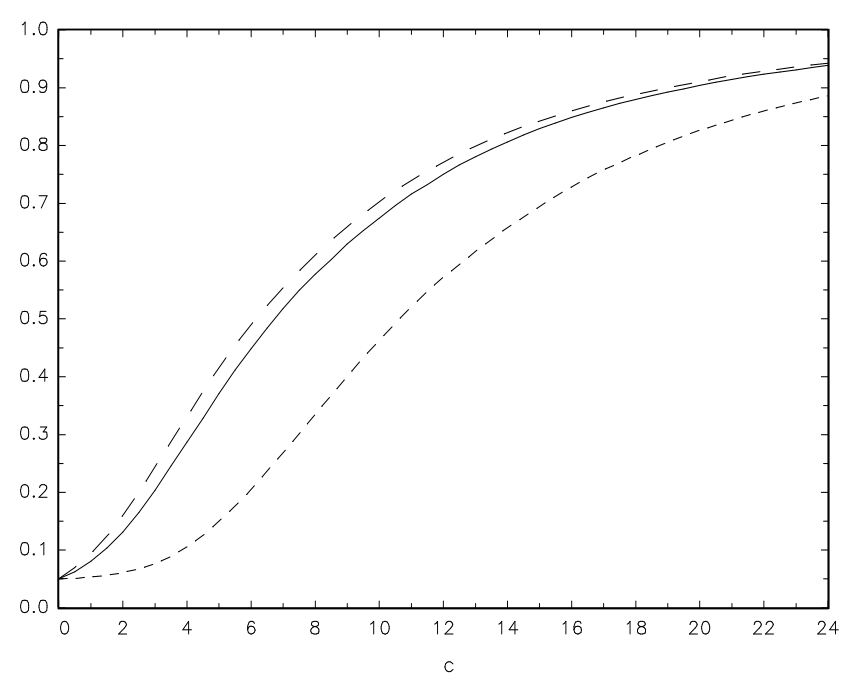

(e) $\left(\tau_{1}, \tau_{2}\right)=(0.90,1.00)$

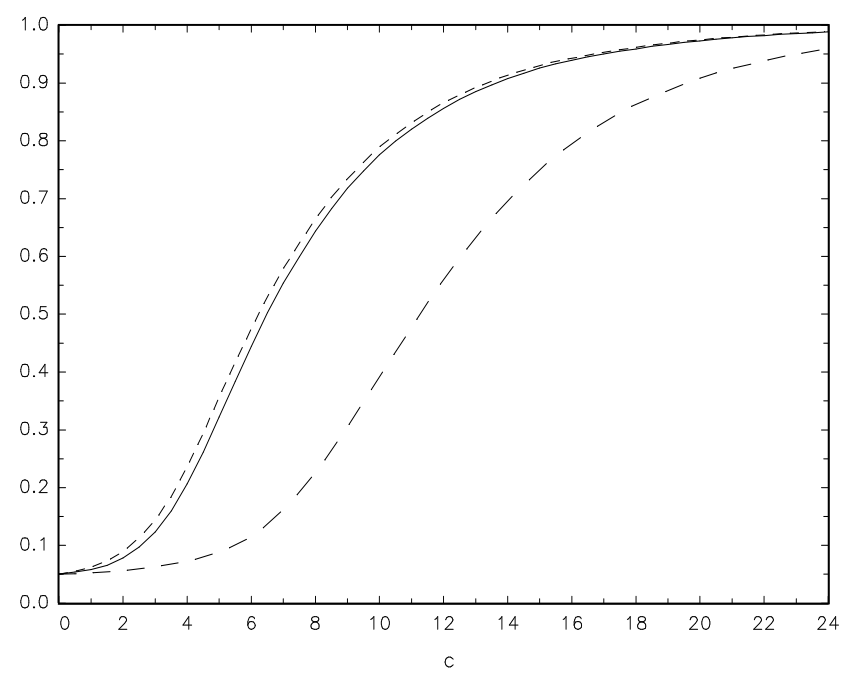

(b) $\left(\tau_{1}, \tau_{2}\right)=(0.40,0.60)$, Case 1

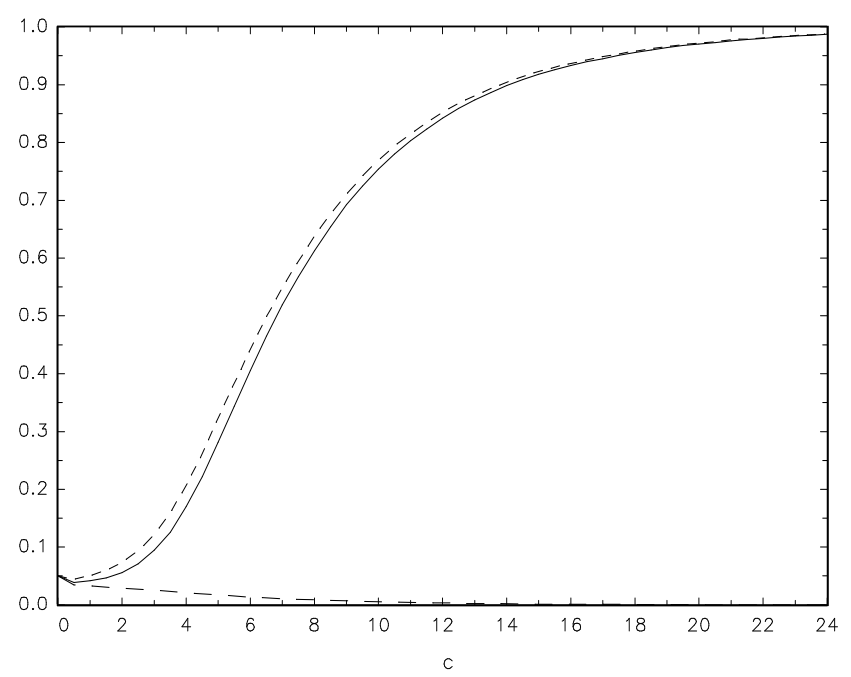

(d) $\left(\tau_{1}, \tau_{2}\right)=(0.40,0.60)$, Case 2

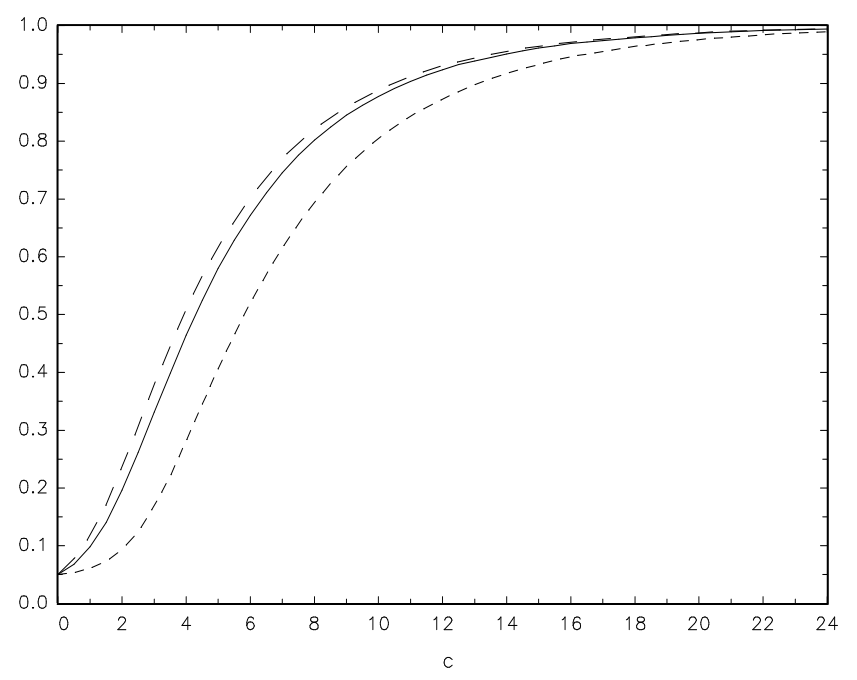

(f) $\left(\tau_{1}, \tau_{2}\right)=(0.80,1.00)$

Figure 1. Local asymptotic power: $P W Y:---, H B:--, U R:-$ 


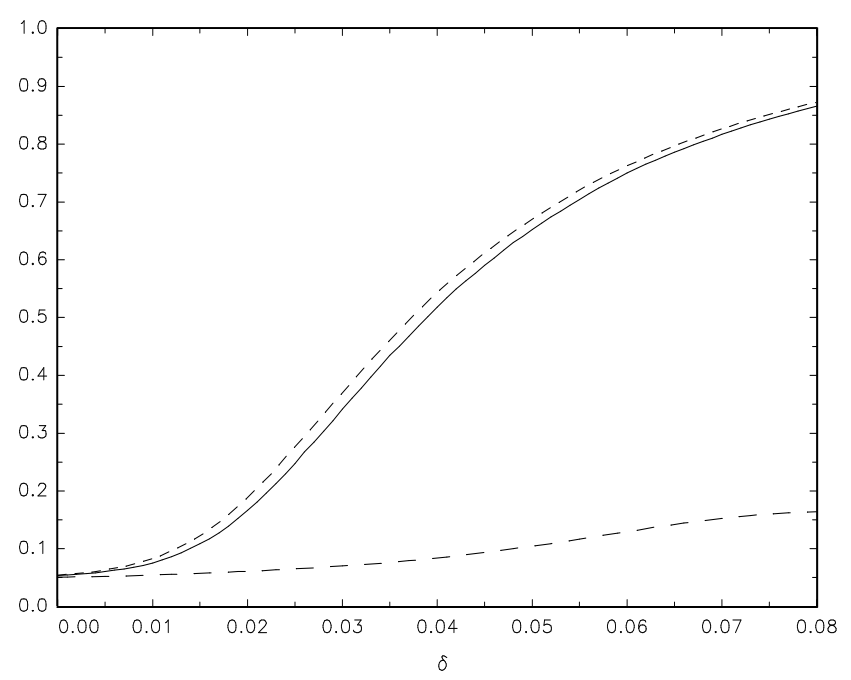

(a) $\left(\tau_{1}, \tau_{2}\right)=(0.45,0.55)$, Case 1

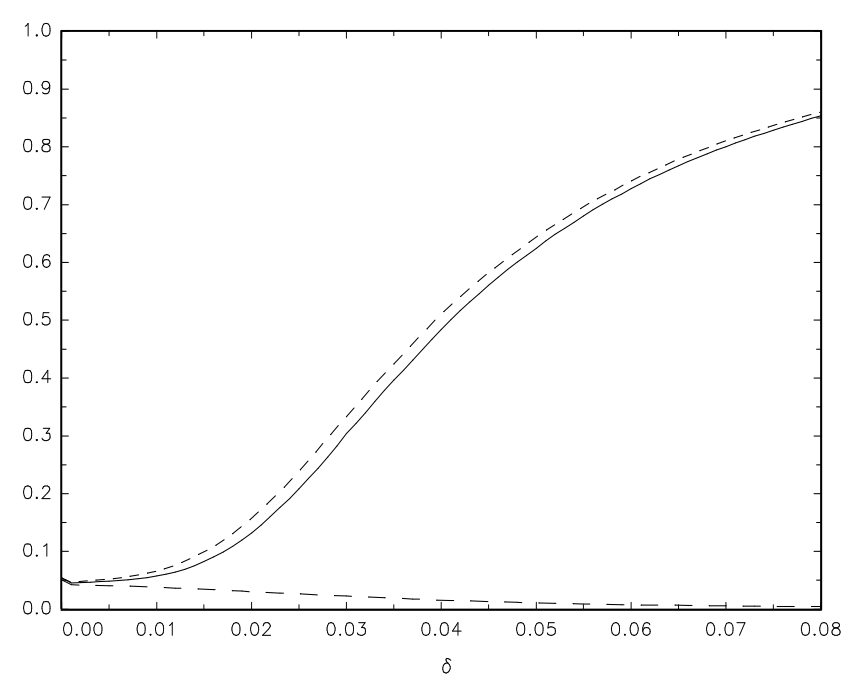

(c) $\left(\tau_{1}, \tau_{2}\right)=(0.45,0.55)$, Case 2

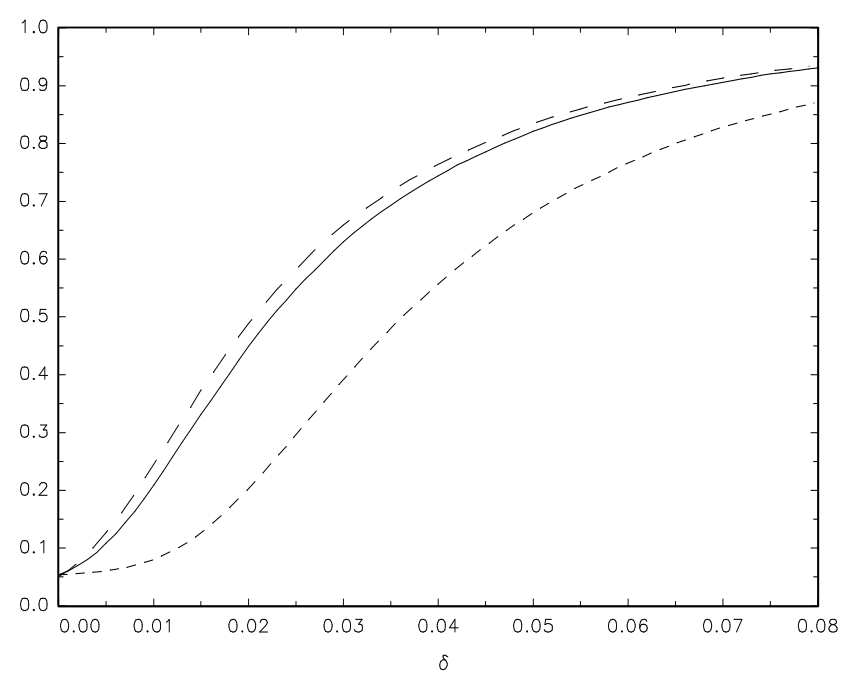

(e) $\left(\tau_{1}, \tau_{2}\right)=(0.90,1.00)$

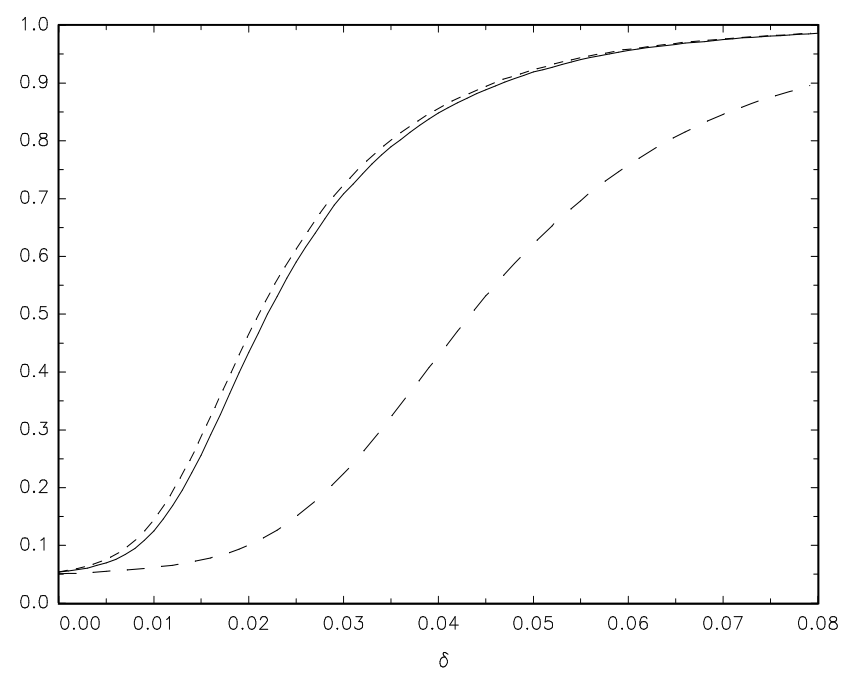

(b) $\left(\tau_{1}, \tau_{2}\right)=(0.40,0.60)$, Case 1

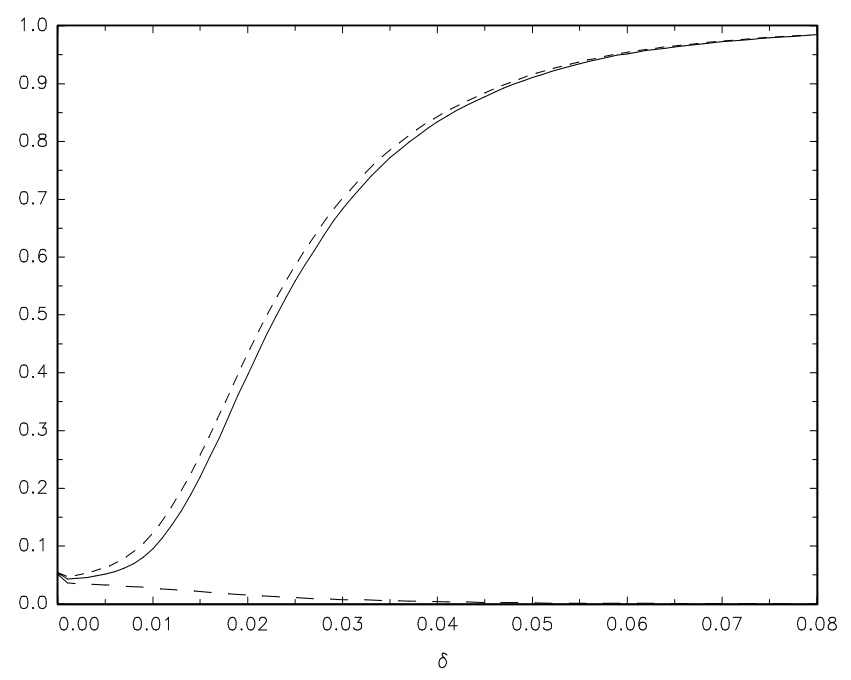

(d) $\left(\tau_{1}, \tau_{2}\right)=(0.40,0.60)$, Case 2

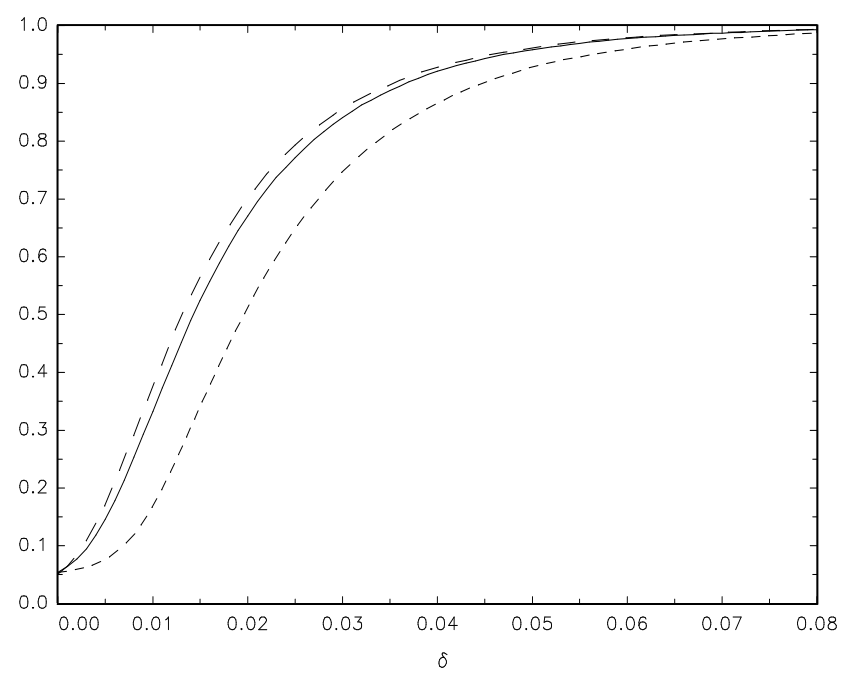

(f) $\left(\tau_{1}, \tau_{2}\right)=(0.80,1.00)$

Figure 2. Finite sample power, $T=300, v_{t} \sim N(0,1): P W Y:---, H B:--, U R:-$ 


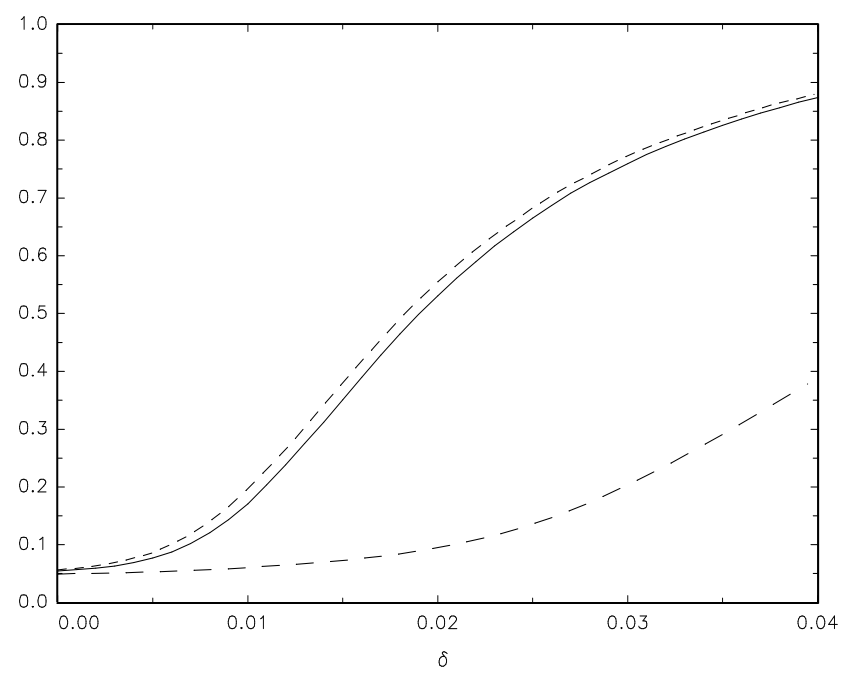

(a) $\left(\tau_{1}, \tau_{2}\right)=(0.45,0.55)$, Case 1

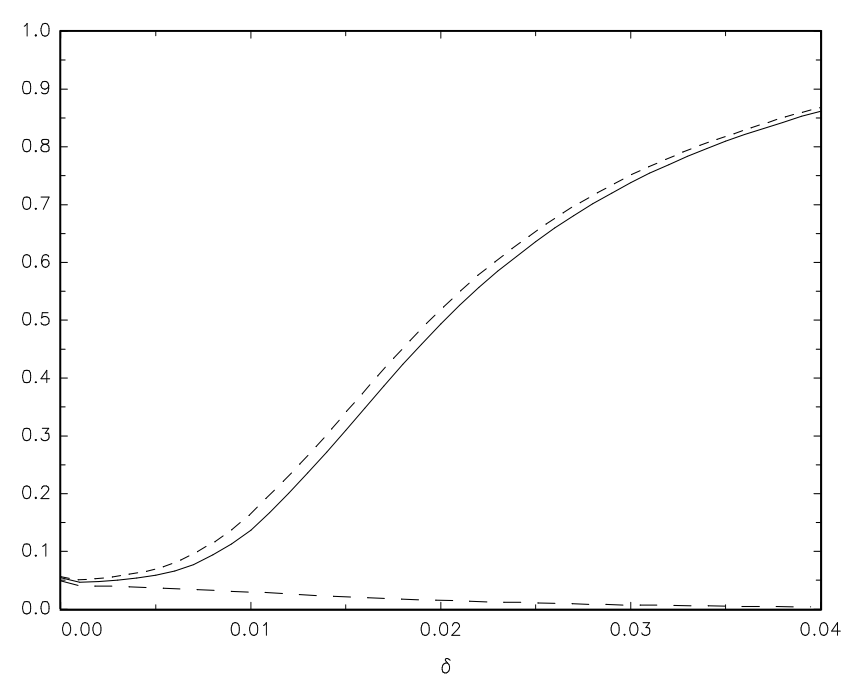

(c) $\left(\tau_{1}, \tau_{2}\right)=(0.45,0.55)$, Case 2

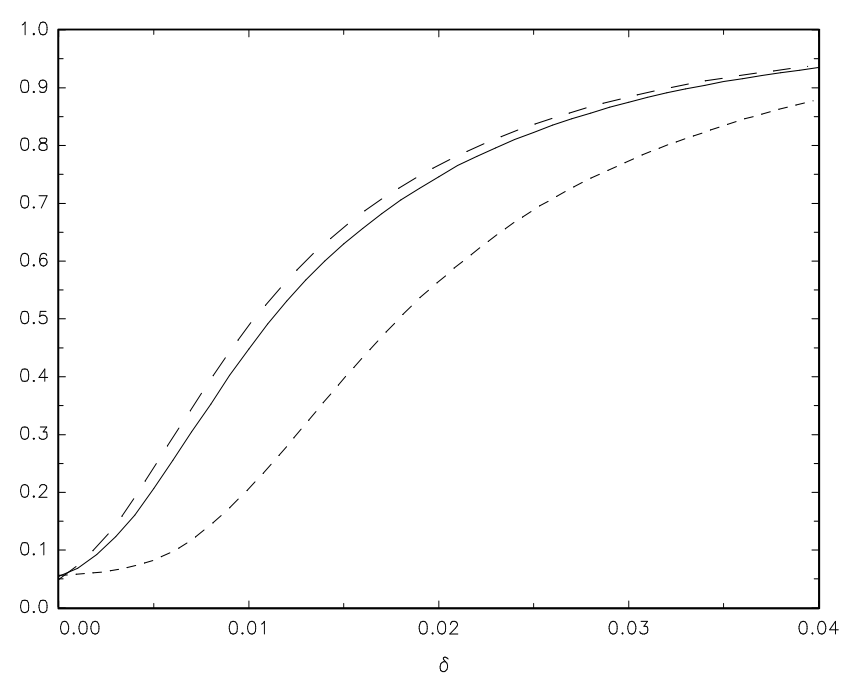

(e) $\left(\tau_{1}, \tau_{2}\right)=(0.90,1.00)$

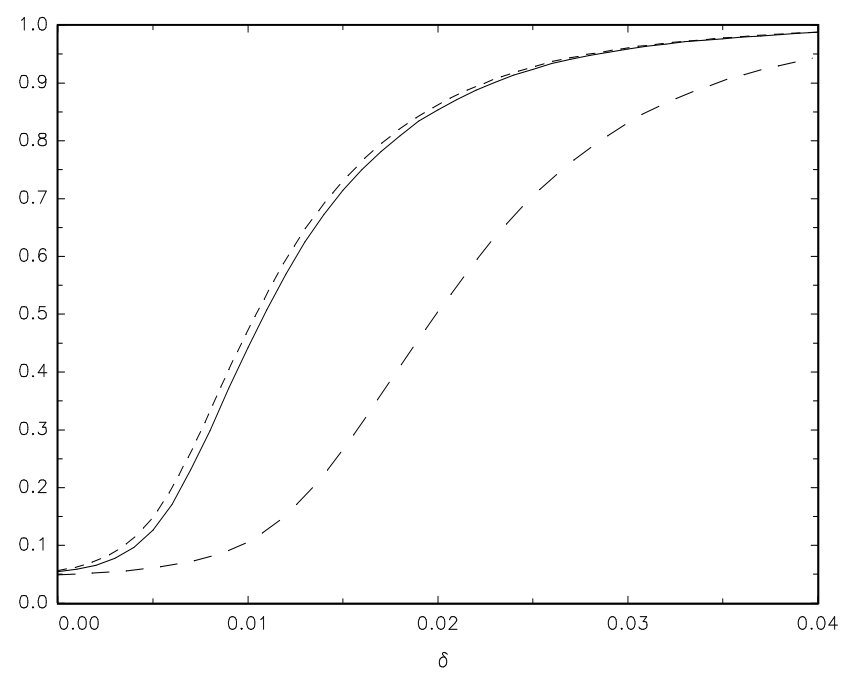

(b) $\left(\tau_{1}, \tau_{2}\right)=(0.40,0.60)$, Case 1

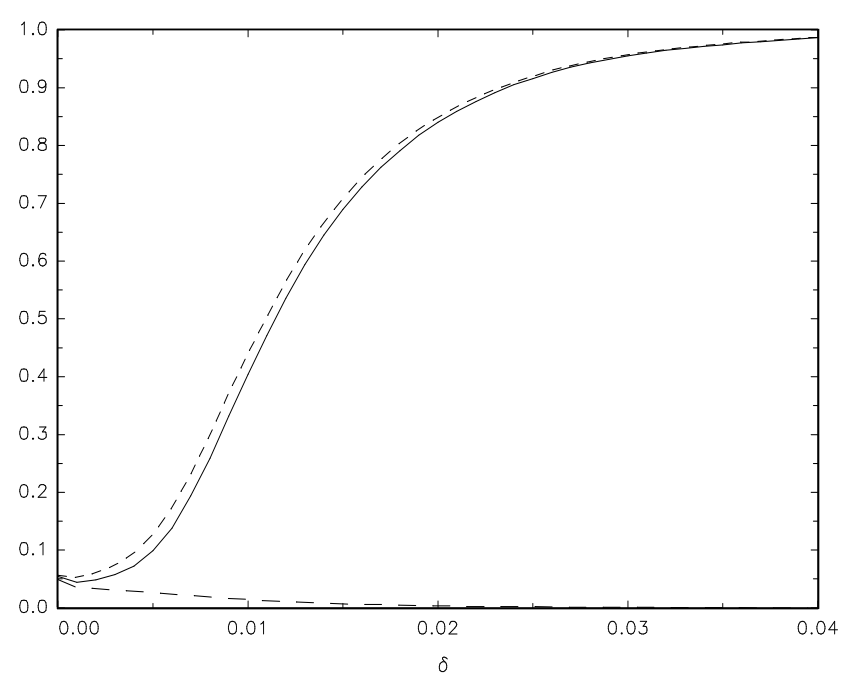

(d) $\left(\tau_{1}, \tau_{2}\right)=(0.40,0.60)$, Case 2

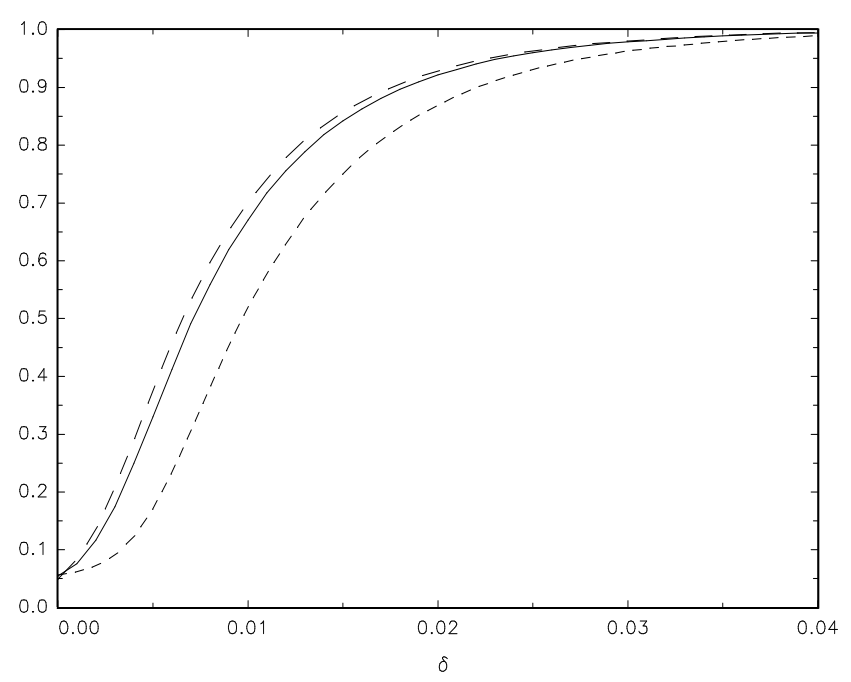

(f) $\left(\tau_{1}, \tau_{2}\right)=(0.80,1.00)$

Figure 3. Finite sample power, $T=600, v_{t} \sim N(0,1): P W Y:---, H B:--, U R:-$ 


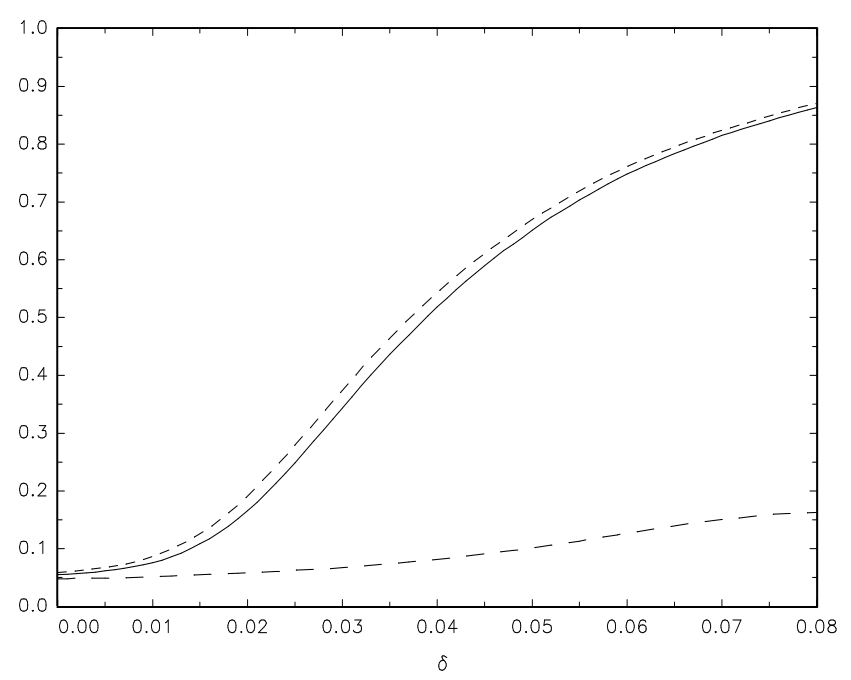

(a) $\left(\tau_{1}, \tau_{2}\right)=(0.45,0.55)$, Case 1

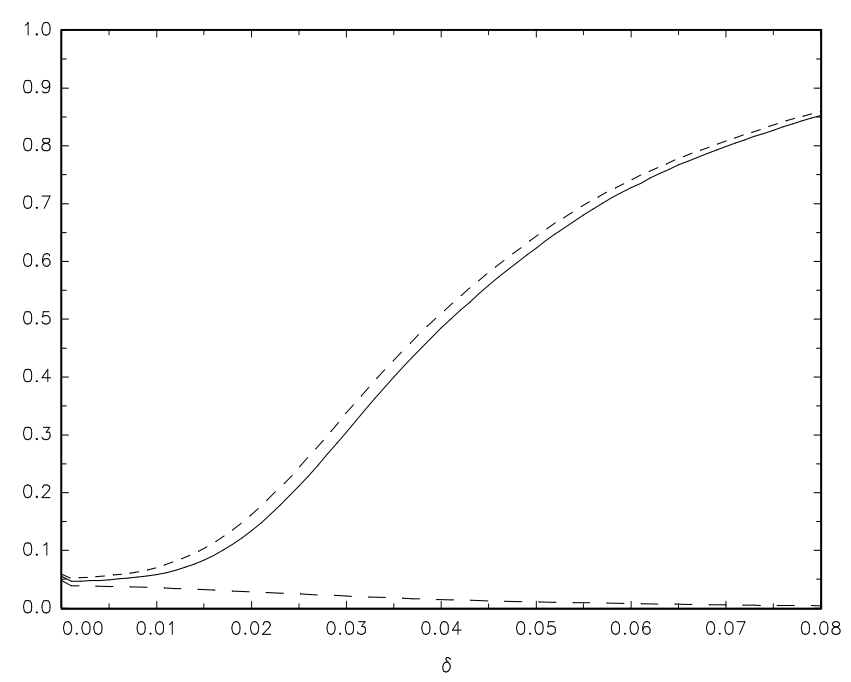

(c) $\left(\tau_{1}, \tau_{2}\right)=(0.45,0.55)$, Case 2

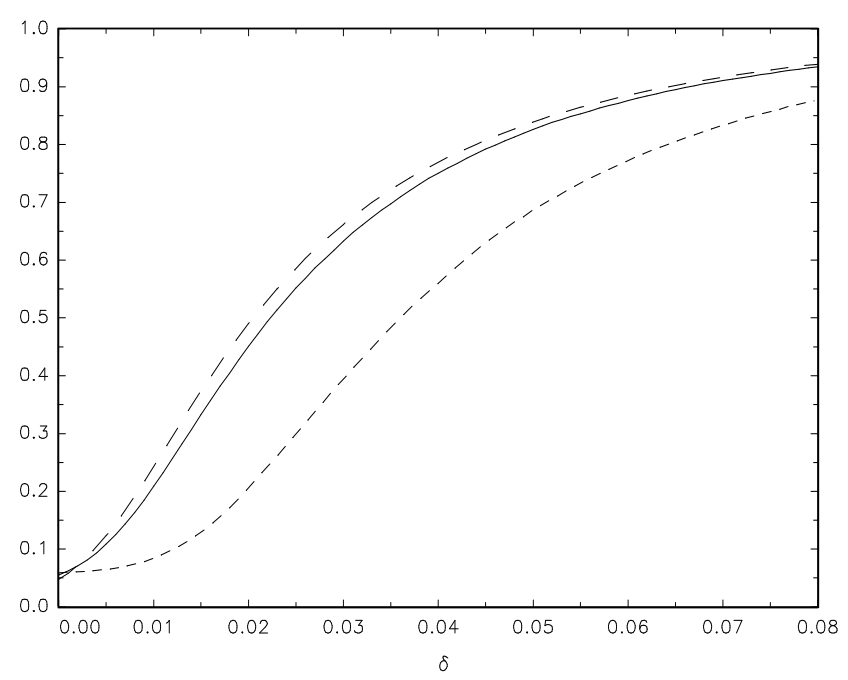

(e) $\left(\tau_{1}, \tau_{2}\right)=(0.90,1.00)$

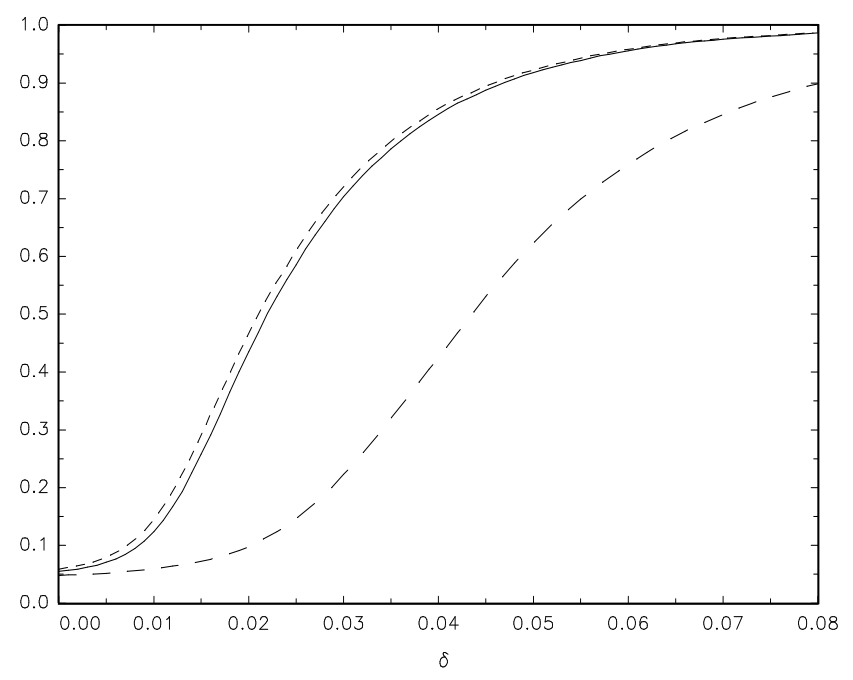

(b) $\left(\tau_{1}, \tau_{2}\right)=(0.40,0.60)$, Case 1

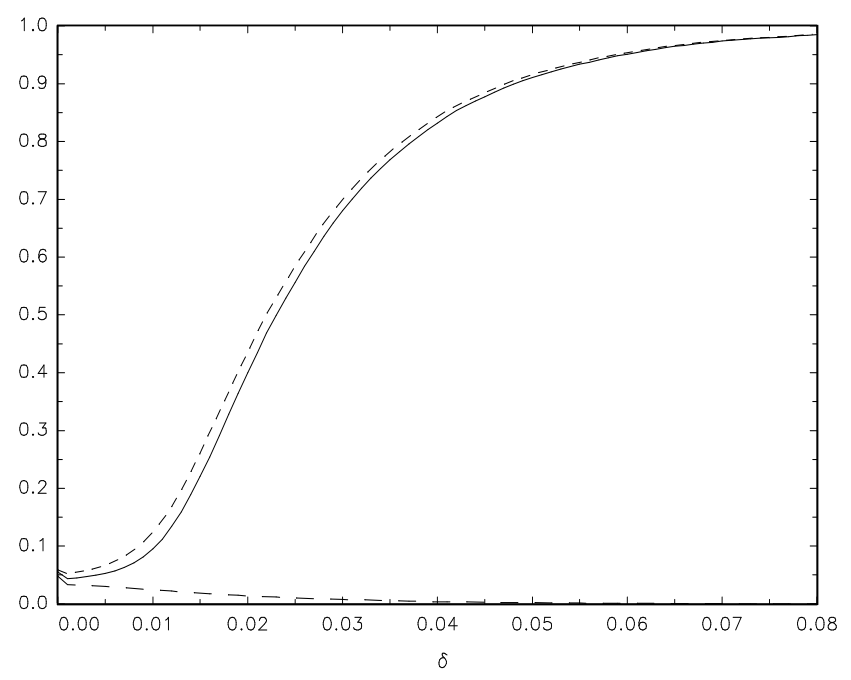

(d) $\left(\tau_{1}, \tau_{2}\right)=(0.40,0.60)$, Case 2

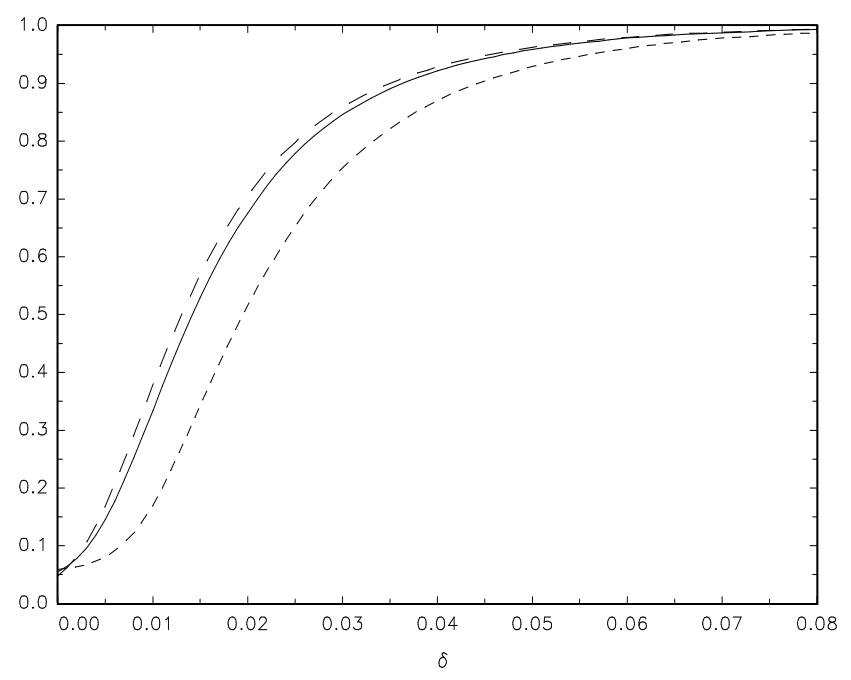

(f) $\left(\tau_{1}, \tau_{2}\right)=(0.80,1.00)$

Figure 4. Finite sample power, $T=300, v_{t} \sim t_{5}: P W Y:---, H B:--, U R:-$ 


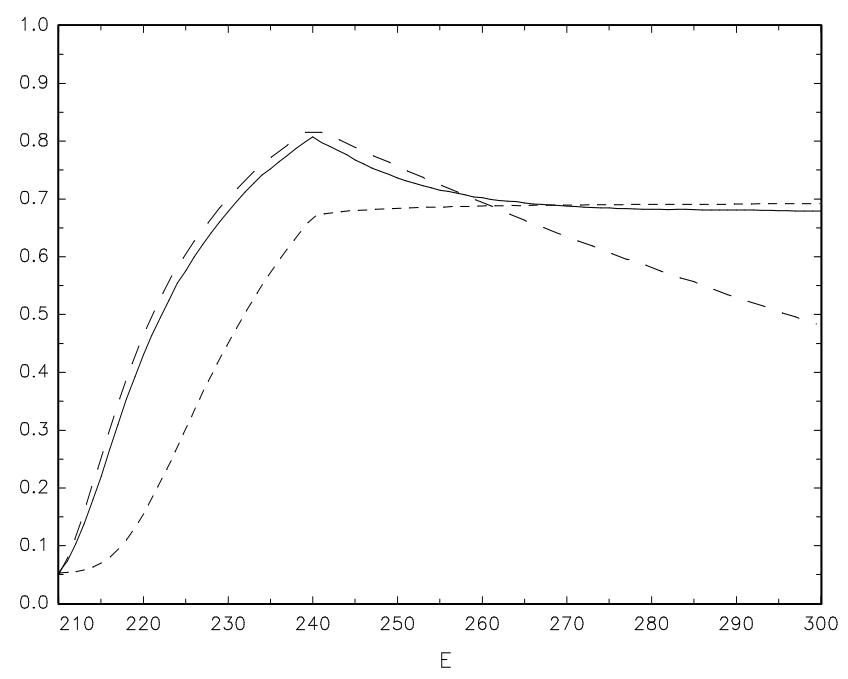

(a) Case 1

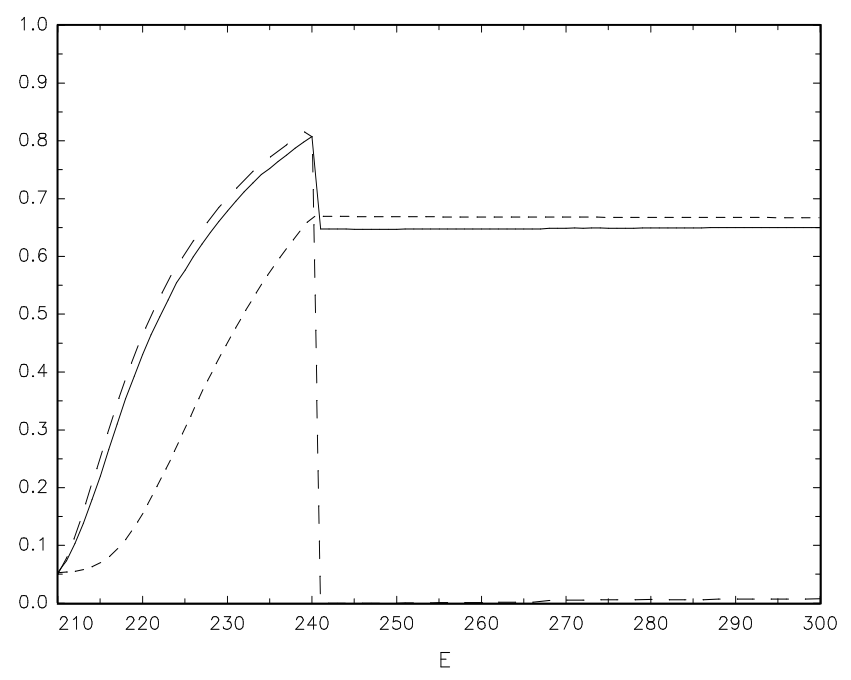

(b) Case 2

Figure 5. Finite sample power across sample end dates, $T=300, \delta=1.05,\left(\tau_{1}, \tau_{2}\right)=(0.70,0.80)$ : $P W Y:--, H B:--, U R:-$

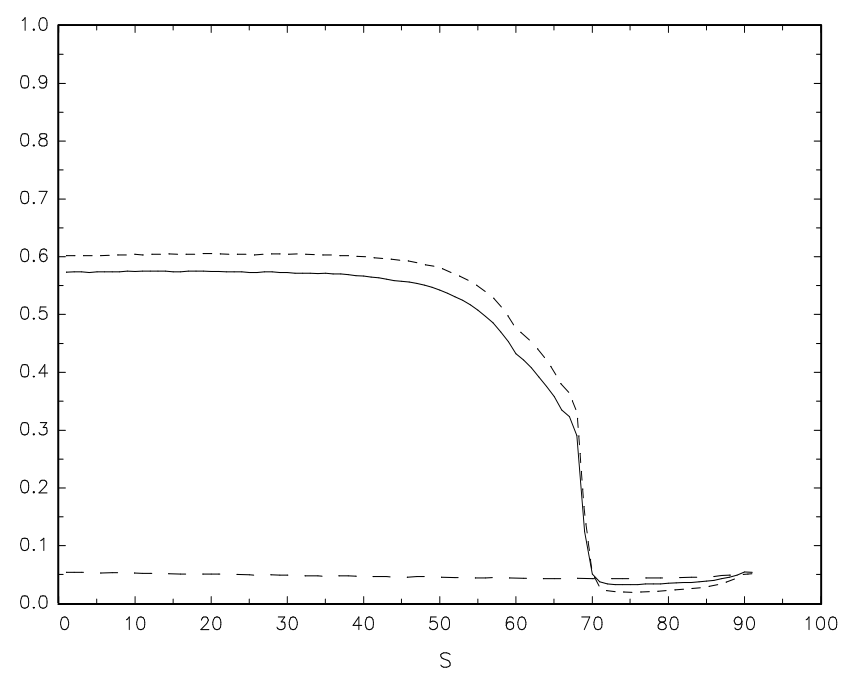

(a) Case 1

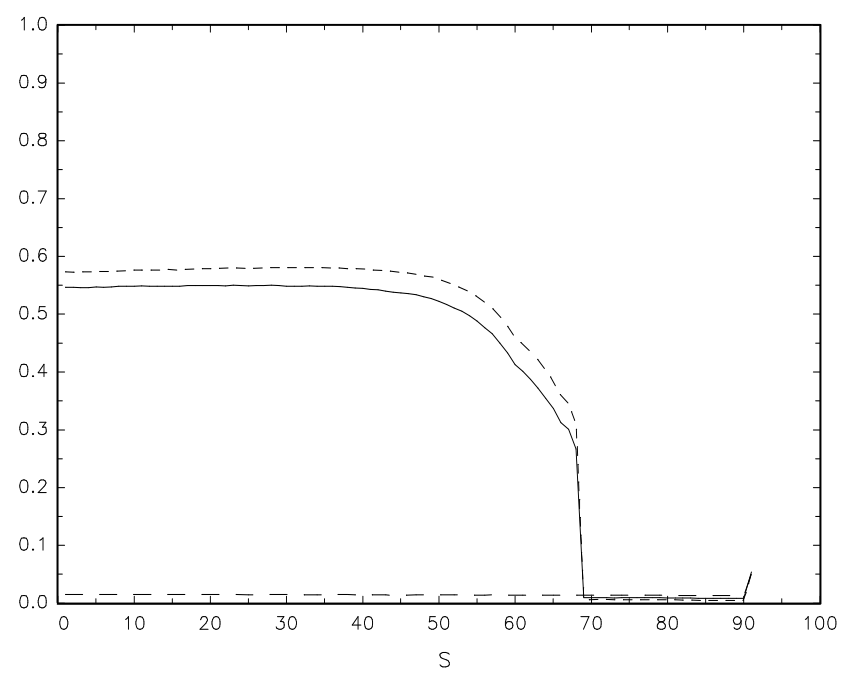

(b) Case 2

Figure 6. Finite sample power across sample start dates, $T=300, \delta=1.05,\left(\tau_{1}, \tau_{2}\right)=(0.20,0.30)$ : $P W Y:--, H B:--, U R:-$ 


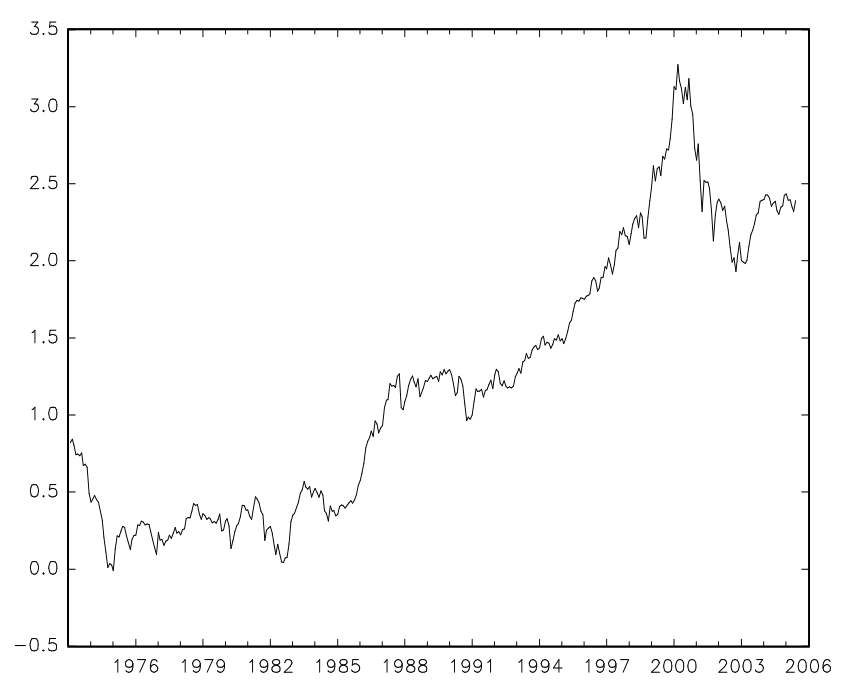

(a) Prices

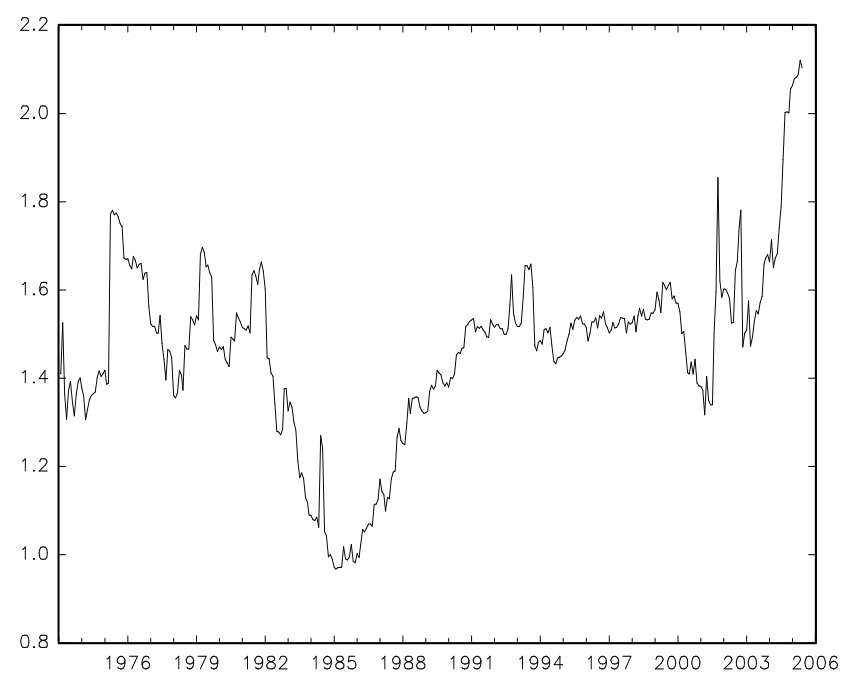

(b) Dividends

Figure 7. Logarithms of Nasdaq composite real price index and real dividends, 1973:2-2005:6

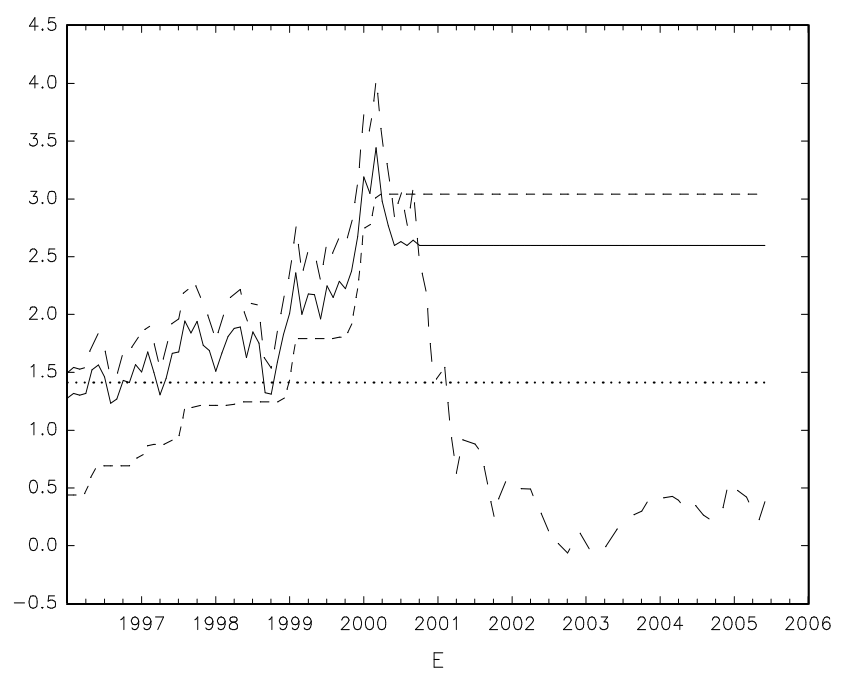

(a) Prices

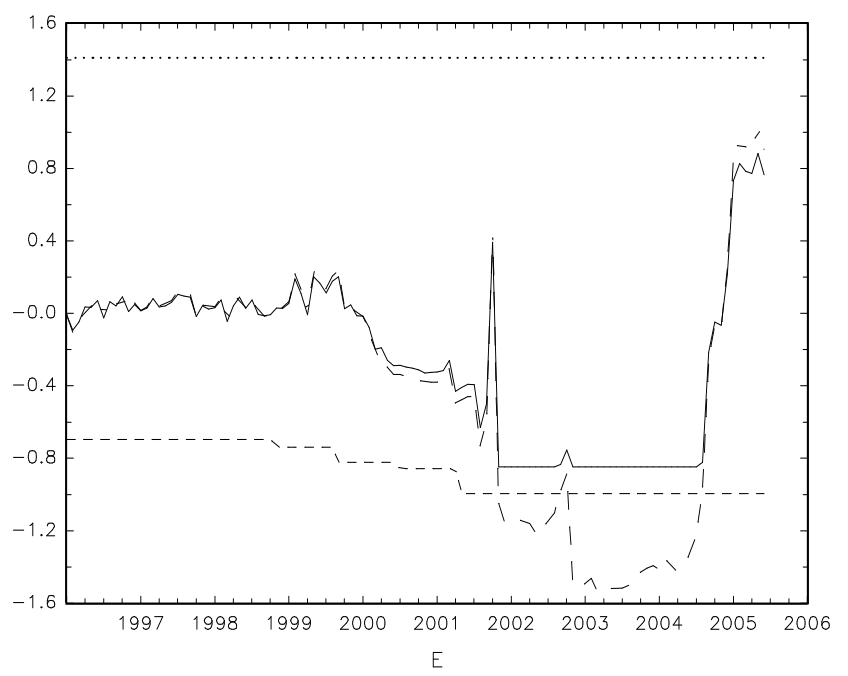

(b) Dividends

Figure 8. Application of tests to Nasdaq composite real price index and real dividends, 1973:2-E: $P W Y:-\cdots, H B:--, U R:-, \cdots: 0.05$-level critical value 